\title{
Florística e caracterização da vegetação da Toca dos Urubus, Baependi, Minas Gerais, Brasil
}

\author{
Fabrício Moreira Ferreira ${ }^{1,3}$ \& Rafaela Campostrini Forzza ${ }^{2}$ \\ ${ }^{1}$ Universidade Estadual de Feira de Santana - UEFS, \\ BR 116, Km 03, CEP 44031-460, Feira de Santana, BH, Brasil \\ ${ }^{2}$ Jardim Botânico do Rio de Janeiro, \\ Pacheco Leão, 915, CEP 22460-030, Rio de Janeiro, RJ, Brasil \\ ${ }^{3}$ Autor para correspondência: fmoreiraf@yahoo.com.br
}

FERREIRA, F.M. \& FORZZA, R.C. Floristics and characterization of the vegetation at Toca dos Urubus, Baependi, Minas Gerais State, Brazil. Biota Neotrop., 9(4): http://www.biotaneotropica.org.br/v9n4/en/abstr act?inventory+bn00909042009.

\begin{abstract}
Floristics and characterization of the vegetation at Toca dos Urubus, Minas Gerais State, Brazil) Toca dos Urubus is located in Baependi municipality, Minas Gerais State, and belong the Alto Rio Grande region. The vegetation comprised a mosaic with cerrado, rocky grassland and seasonally dry tropical forest. A floristic survey was undertaking, restrict to vascular plants, and a descriptive characterization of vegetation is presented. A total of 403 species distributed in 260 genus and 77 families were sampled. Angiosperms comprised 395 species, while pteridophytes comprised eight species. The richest families were Asteraceae (66 spp.), Poaceae (52 spp.), Fabaceae (32 spp.), Cyperaceae (19 spp.), Melastomataceae and Rubiaceae (16 spp.). Most of the species (322 spp.) occur on cerrado, while 247 occur on rocky grassland, and 71 species on seasonally dry tropical forest. Despite the small area studied, approximately 25 ha, high species diversity was found, and 10 of these are included at the Red List of Minas Gerais, strengthening the need for conservation planning within the region.

Keywords: flora, rocky grasslands, cerrado, seasonally dry tropical forest, Mantiqueira Range.
\end{abstract}

FERREIRA, F.M. \& FORZZA, R.C. Florística e caracterização da vegetação da Toca dos Urubus, Baependi, Minas Gerais, Brasil. Biota Neotrop., 9(4): http://www.biotaneotropica.org.br/v9n4/pt/abstract?inventory+bn 00909042009.

Resumo: (Florística e caracterização da vegetação da Toca dos Urubus, Baependi, Minas Gerais, Brasil) A Toca dos Urubus localiza-se no município de Baependi, sul de Minas Gerais, e faz parte da região do Alto Rio Grande. Sua cobertura vegetal compreende um mosaico formado por cerrado, campo rupestre e floresta estacional semidecidual. Foi realizado um levantamento florístico, restrito às espécies vasculares, e uma descrição das três fisionomias encontradas na área. Foram registradas 403 espécies (395 angiospermas e 8 pteridófitas) distribuídas em 77 famílias e 260 gêneros. As famílias mais ricas em número de espécies foram Asteraceae (66 spp.), Poaceae (52 spp.), Fabaceae (32 spp.), Cyperaceae (19 spp.), Melastomataceae e Rubiaceae (16 spp.). O cerrado apresentou o maior número de espécies (322 spp.), seguido pelo campo rupestre $(247 \mathrm{spp}$.) e pelo fragmento de floresta estacional (71 spp.). Apesar da pequena área abordada no presente estudo, aproximadamente 25 ha, uma grande riqueza de espécies foi encontrada, dentre estas 10 são citadas na Lista Vermelha das Espécies Ameaçadas de Extinção da Flora de Minas Gerais, o que reforça ainda mais a necessidade de conservação dessa região.

Palavras-chave: flora, campo rupestre, cerrado, floresta estacional semidecidual, Serra da Mantiqueira. 


\section{Introdução}

A Serra da Mantiqueira estende-se pelo leste do estado de São Paulo, sul de Minas Gerais e sudoeste do Rio de Janeiro, atuando como um divisor de águas entre as bacias do Rio Grande, em Minas Gerais, e Paraíba do Sul, em São Paulo e Rio de Janeiro (Braga \& Andrade 2005). Seu relevo é formado por escarpas elevadas e morros, nos quais o planalto mineiro termina diante do Vale do Paraíba, e encontra-se dividido nesta zona em duas porções: Serra da Mantiqueira Oriental e Serra da Mantiqueira Ocidental (Ponçano et al. 1981). No interior da porção ocidental da Mantiqueira, em direção norte, encontra-se o Planalto Sul de Minas Gerais ou região do Alto Rio Grande, onde se localizam importantes nascentes e afluentes desse rio (Silva 2005). A topografia da região do Alto Rio Grande é predominantemente montanhosa com altitudes variando, em sua maior parte, entre 700 e $1.400 \mathrm{~m}$ acima do nível do mar (Oliveira Filho et al. 1994a).

A vegetação primária da região do Alto Rio Grande compreendia um extenso mosaico vegetacional composto por manchas florestais, cerrados, campos rupestres e campos de altitude (Azevedo 1962, IBGE 1993). Tal diversidade de formações deve-se, principalmente, ao relevo fortemente acidentado e ao fato de a região abrigar uma das transições entre os cerrados do Brasil Central e as florestas semideciduais do sudeste e sul do país (Oliveira Filho \& Fluminhan-Filho 1999). Neste contexto, as fisionomias de campo rupestre e campo de altitude no sul de Minas Gerais estão associadas a solos rasos e jovens do topo das montanhas, enquanto o cerrado e florestas semideciduais compartilham solos mais antigos e profundos, sendo as florestas encontradas em locais de solos mais férteis (Eiten 1982).

As primeiras tentativas de ocupação da região do Alto Rio Grande aconteceram nos séculos XVII e XVIII, com a entrada das bandeiras paulistas em busca do ouro (Gomes 2005). Posteriormente, foi a extração de madeira e a agropecuária que, realmente, levaram à ocupação da área. Em consequiência, houve maciça exploração das terras e drástica redução da vegetação nativa para formação de áreas cultiváveis e atividades mineradoras (Silva 2005).

Com o intuito de proteger essa importante e complexa área, foi criada em 1985, pelo decreto 91.304, a Área de Proteção Ambiental da Serra da Mantiqueira (APA Mantiqueira), que abrange uma área de 422.873 ha de 25 municípios nos estados de São Paulo, Minas Gerais e Rio de Janeiro (Ribeiro 2005). A importância da região foi ressaltada quando a região do Alto Rio Grande foi considerada área de importância biológica especial e prioritária para a conservação da biodiversidade em Minas Gerais, devido à alta riqueza de espécies da fauna e flora (Drummond et al. 2005).

Embora nos últimos anos o número de estudos sobre a vegetação na Mantiqueira tenha aumentado (Botrel et al. 2002, Carvalho et al. 1995a, Gavilanes et al. 1992a, b, Oliveira Filho et al. 1994a, b, c, d, 2004, Rodrigues et al. 2003, Souza et al. 2003, Vilela et al. 1995, 2000, van den Berg \& Oliveira Filho 2000), estes são, na grande maioria, restritos às formações florestais, enquanto as áreas campestres continuam pouco amostradas. Assim, este estudo tem por objetivo contribuir para o conhecimento da vegetação da região do Alto Rio Grande apresentando a lista de espécies registradas na Toca dos Urubus, uma área predominantemente de vegetação campestre do município de Baependi, bem como fornecer uma caracterização das três fisionomias encontradas na área.

\section{Material e Métodos}

O município de Baependi localiza-se na microrregião do Circuito das Águas no sul de Minas Gerais (Figura 1) e, de acordo com o Sistema Estadual de Gerenciamento de Recursos Hídricos, está
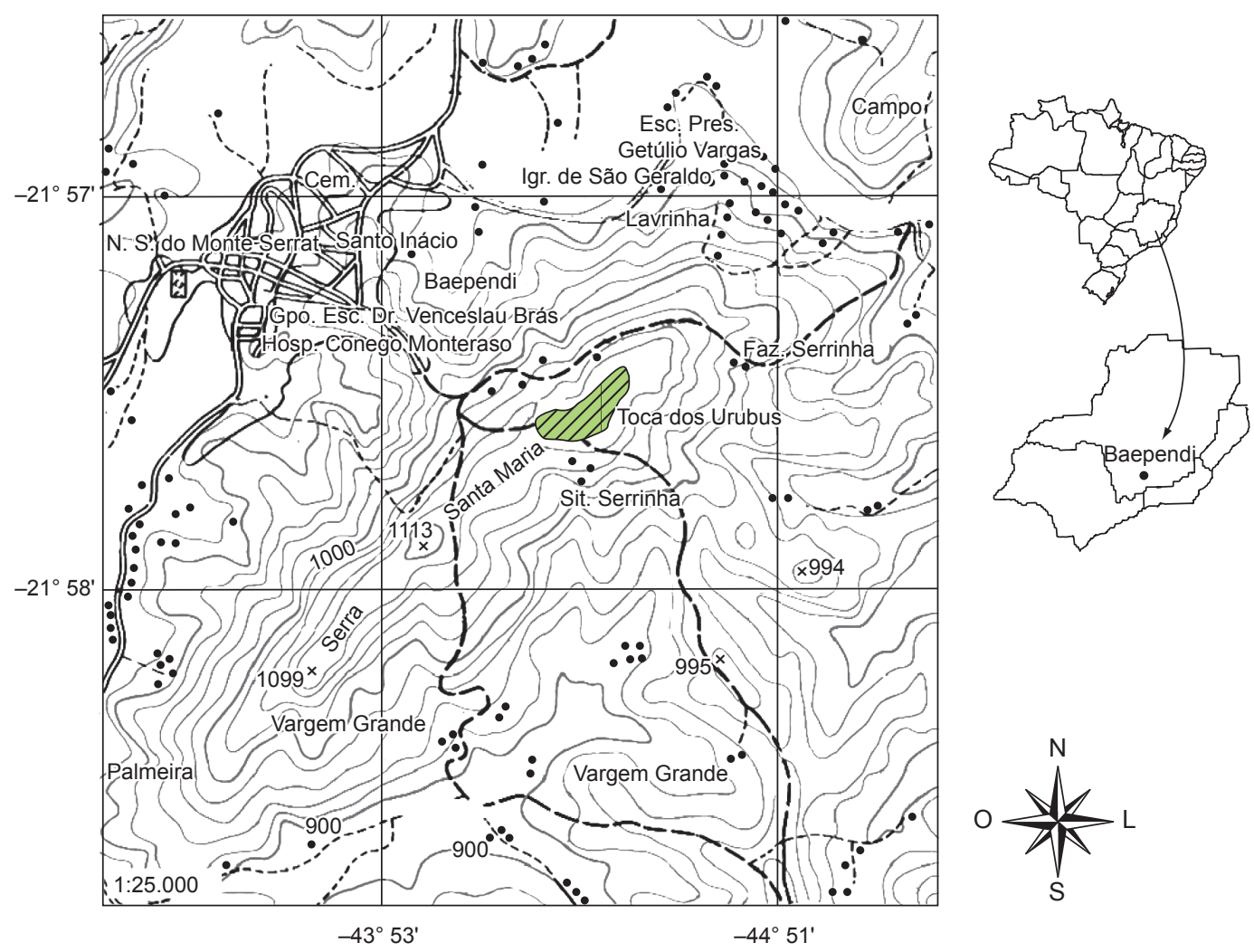

Figura 1. Localização da Toca dos Urubus, Baependi (MG, Brasil).

Figure1. The localization of the Toca dos Urubus, Baependi (MG, Brazil). 


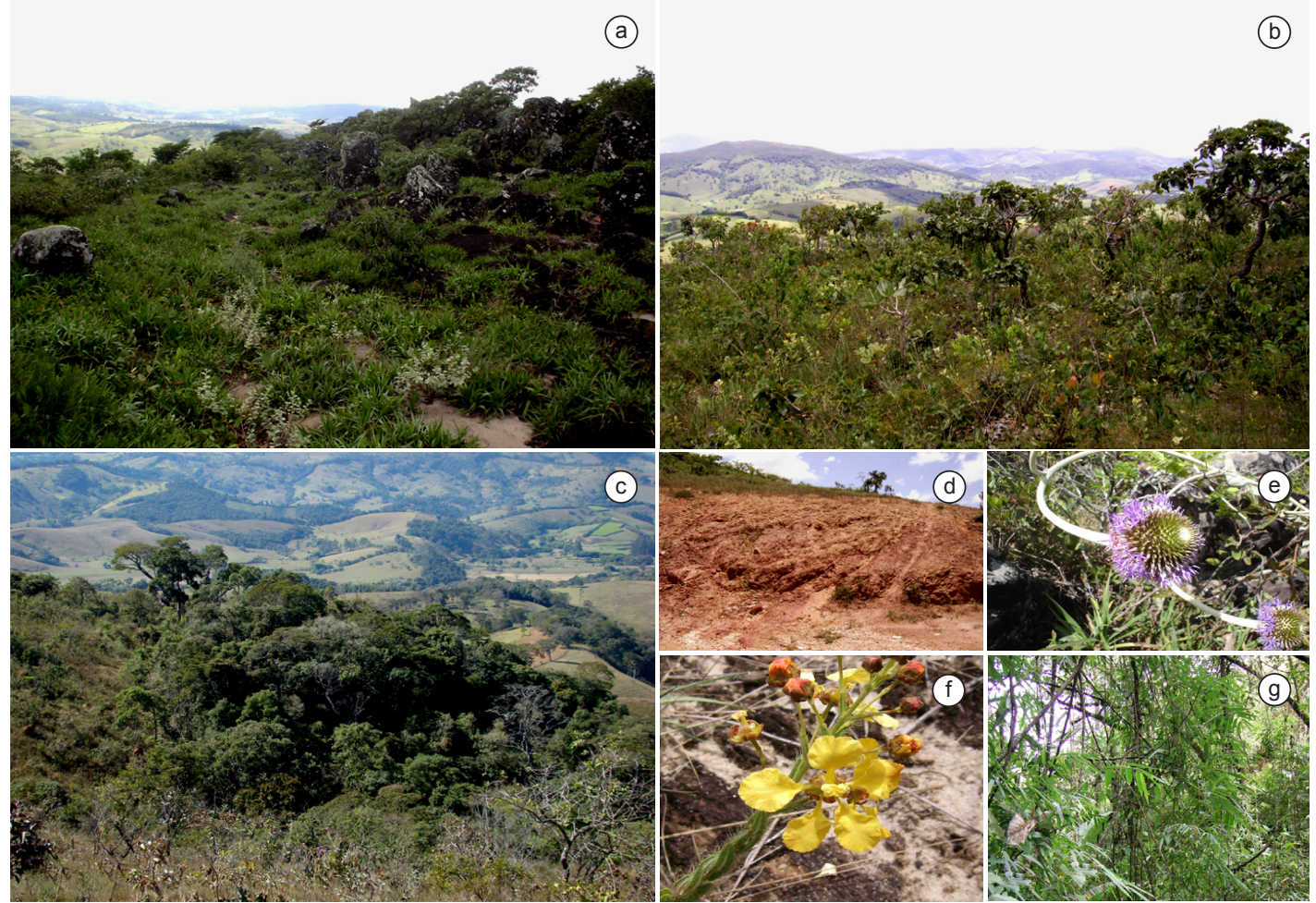

Figura 2. Diferentes fisionomias encontradas na Toca dos Urubus, Baependi. a) Campo rupestre. b) Cerrado. c) Floresta Estacional Semidecidual Submontana. d) Erosão causada pela retirada da cobertura vegetal. e) Chresta sphaerocephala DC. f) Camarea hyrsuta A. St.-Hil. g) Chusquea tenuiglumis Döll apresenta hábito escandente e cobre grande área no fragmento de floresta semidecidual.

Figure 2. Different physiognomies found in the Toca dos Urubus, Baependi. a) Rocky Grassland. b) Cerrado. c) Seasonally Dry Tropical Forest. d) Erosion due to the vegetal cover withdrawal. e) Chresta sphaerocephala DC. f) Camarea hyrsuta A. St.-Hil. g) Chusquea tenuiglumis Döll, exhibits a clambering habit, and covers large areas of fragment of semideciduous forest.

inserido na região Alto Rio Grande (Viola 2008) (Figura 1). Segundo a classificação de Köppen, possui clima Cwb (tropical de altitude) com verões quentes e úmidos e invernos frios e secos (Minas Gerais 1982). A temperatura média anual varia entre 18 e $19^{\circ} \mathrm{C}$ e a média pluviométrica é de $1.400 \mathrm{~mm}$, com chuvas mais concentradas entre dezembro e março, e os meses de junho, julho e agosto compreendendo o período seco (Minas Gerais 1982).

A Toca dos Urubus é uma localidade situada no extremo norte da Serra de Santa Maria do Baependi que corre no sentido NO/SE a partir do ponto $21^{\circ} 58^{\prime} \mathrm{S}$ e $44^{\circ} 52^{\prime} 26^{\prime \prime} \mathrm{W}$, com cerca de $6 \mathrm{~km}$ de extensão, e atua como divisor de águas do Rio Baependi e Ribeirão Palmeira, tributários do Rio Verde, afluente do Rio Grande. Apesar de pertencer à comarca de Baependi, ou seja, ao município, a área não possui nenhuma unidade de conservação e está rodeada por fazendas particulares. Sua cobertura vegetal compreende um mosaico onde predominam as fisionomias de cerrado e campo rupestre. Além das formações campestres, manchas de florestas estacionais são encontradas nos vales ou grotas onde há maior umidade devido à convergência das bacias de drenagem do alto da serra.

O levantamento florístico se restringiu a uma área de aproximadamente 25 ha onde as três fisionomias ocorrentes na serra são mais preservadas. As campanhas de campo foram realizadas durante todos os meses do ano, de março de 2002 a abril de 2006 e cobriram toda área. Os espécimes coletados foram incorporados aos herbários CESJ, HUEFS, R e RB (acrônimos segundo Holmgren et al. 1990). As espécies foram identificadas por meio de bibliografia específica, comparação com espécimes já determinados nos herbários supracitados e através da contribuição de especialistas. Apenas 18 táxons (ca. $4,4 \%$ ) foram identificados somente até gênero. Os dados sobre o ambiente onde ocorrem as espécies e hábito foram baseados em observações de campo.

Foram consideradas ervas as espécies não lenhosas tanto terrestres quanto rupícolas ou epífitas. Espécies terrestres lenhosas, inclusive as xilopodiosas, predominantemente menores que $30 \mathrm{~cm}$, foram denominadas subarbustos; as que, em geral, se enquadravam entre $30 \mathrm{~cm}$ e $1 \mathrm{~m}$ de altura foram denominadas arbustos. Para a categorização das espécies arbóreas utilizou-se o Catálogo das Árvores Nativas de Minas Gerais (Oliveira Filho 2006). Espécies reptantes ou trepadeiras, lenhosas ou não, foram classificadas como lianas. Espécies com hábito escandente foram classificadas na categoria de ervas escandentes, tanto as herbáceas quanto as lenhosas. Foram consideradas espécies ruderais aquelas citadas por Lorenzi (1991).

Determinou-se, para a área de estudo, três fisionomias: Cerrado (sensu Veloso et al. 1991), Floresta Estacional Semidecidual Montana (FESM) (sensu Veloso et al. 1991) e Campo Rupestre (sensu Joly 1970, Harley 1995, Giulietti et al. 1987) (Figura 2a, b, c).

\section{Resultados}

Foram registradas na área 403 espécies de plantas vasculares. As angiospermas compreenderam 395 espécies agrupadas em 253 gêneros e 72 famílias. As pteridófitas foram representadas na área por oito espécies pertencentes a sete gêneros e cinco famílias (Tabela 1). Asteraceae foi a família mais rica em número de espécies (66 spp.), seguida por Poaceae (52 spp.), Fabaceae (32 spp.), Cyperaceae (19 spp.), Melastomataceae e Rubiaceae (16 spp.), 
Tabela 1. Lista das espécies vasculares ocorrentes na Toca dos Urubus, Baependi, Minas Gerais. "Status" indica o grau de ameaça segundo Drummond et al. (2008) (EP: Em Perigo; VU: Vulnerável; QA: Quase Ameaçada; DD: Dados Deficientes) e Oliveira Filho (2006) (M.Rara: muito rara; Rara: rara; Rss.: raríssima). Abreviação dos tipos de vegetação: C: cerrado; R: campo rupestre; M: mata estacional. Hábito: AB: arbusto; AV: árvore; AV/SB: árvore/subarbusto; EE: erva escandente; EP: erva epífita; ER: erva rupícola; ET: erva terrestre; LI: liana; SB: subarbusto. FM representa os números de coleta de Fabrício Moreira Ferreira e RC os de Rafaela Campostrini Forzza. As espécies assinaladas com um asterisco (*) são consideradas ruderais segundo Lorenzi (1991).

Table 1. Species list of the vascular plants found in Toca dos Urubus, Baependi, Minas Gerais State. "Status" indicate the conservation status according Drummond et al. (2008) (EP: endangered; VU: vulnerable; QA: near threatened; DD: data deficient) and Oliveira Filho (2006) (M.Rara: very rare; Rara: rare; Rss.: rarely). Abbreviatoins for vegetation types are C: cerrado, R: campo rupestre e M: mata estacional. Abbreviations for life forms are AB: shrub, AV: Tree, AV/SB: tree/subshrub, EE: clambering herb, EP: epiphyte, ER: rupicola herb, ET: herb, LI: vine, SB: subshrub. Abbreviations for collectos are FM: Fabricio Moreira Ferreira, and RC: Rafaela Campostrini Forzza. The asterisk after the species names highlights the weed according Lorenzi (1991).

\begin{tabular}{|c|c|c|c|c|c|c|}
\hline \multicolumn{7}{|c|}{ Angiospermas } \\
\hline Família/Espécie & Status & $\mathbf{C}$ & $\mathbf{R}$ & M & Hábito & Voucher \\
\hline \multicolumn{7}{|l|}{ ACANTHACEAE } \\
\hline Ruellia geminiflora Kunth & - & $\mathrm{x}$ & $\mathrm{x}$ & - & SB & FM 763, 1178 \\
\hline \multicolumn{7}{|l|}{ ALSTROEMERIACEAE } \\
\hline Alstroemeria plantaginea Mart. ex Schult. \& Schult. f. & $\mathrm{EP}$ & $\mathrm{x}$ & - & - & ET & FM 903 \\
\hline \multicolumn{7}{|l|}{ AMARANTHACEAE } \\
\hline Gomphrena virgata Mart. & - & $\mathrm{x}$ & $\mathrm{x}$ & - & SB & FM 177, 859 \\
\hline Pfaffia jubata Mart. & - & - & $\mathrm{x}$ & - & SB & FM 655 \\
\hline \multicolumn{7}{|l|}{ ANACARDIACEAE } \\
\hline Anacardium humile A. St.-Hil. & - & $\mathrm{x}$ & - & - & SB & FM 279, 314 \\
\hline Tapirira obtusa (Benth.) J.D. Mitch. & - & - & - & $\mathrm{x}$ & $\mathrm{AV}$ & FM 1163 \\
\hline \multicolumn{7}{|l|}{ ANNONACEAE } \\
\hline Annona tomentosa F.E. Fr. & - & $\mathrm{x}$ & $\mathrm{x}$ & - & $\mathrm{AB}$ & FM 339, 462 \\
\hline Guatteria australis A. St.-Hil. & - & - & - & $\mathrm{x}$ & $\mathrm{AV}$ & FM 463, 1177 \\
\hline \multicolumn{7}{|l|}{ APIACEAE } \\
\hline Eryngium pristis Cham. \& Schltd. & - & $\mathrm{x}$ & - & - & ET & FM 801 \\
\hline \multicolumn{7}{|l|}{ APOCYNACEAE } \\
\hline Asclepias curassavica L.* & - & $\mathrm{x}$ & - & - & ET & FM 456 \\
\hline Aspidosperma parvifolium A. DC. & M.Rara & $\mathrm{x}$ & - & $\mathrm{x}$ & AV & FM 432, 837, 1169 \\
\hline Aspidosperma tomentosum Mart. & - & $\mathrm{x}$ & $\mathrm{x}$ & - & AV & FM 168, 308, 313 \\
\hline Barjonia erecta (Vell.) K. Schum. & - & $\mathrm{x}$ & $\mathrm{x}$ & - & ET & FM 649 \\
\hline Blepharodon nitidum (Vell.) J.F. Macbr. & - & $\mathrm{x}$ & - & - & LI & FM 676 \\
\hline Forsteronia velloziana (A. DC. ) Woodson & - & - & $\mathrm{x}$ & $\mathrm{x}$ & LI & FM 752, 1181 \\
\hline Hancornia speciosa Gomes & - & $\mathrm{x}$ & - & - & AV & FM 281, 427 \\
\hline Mandevilla pohliana (Stadelm.) A.H. Gentry & - & $\mathrm{x}$ & - & - & ET & FM 305, 306, 619 \\
\hline Minaria acerosa (Mart.) T.U. Kono \& A. Rapini & - & $\mathrm{x}$ & $\mathrm{x}$ & - & SB & FM 660 \\
\hline Oxypetalum strictum Mart. & - & $\mathrm{x}$ & $\mathrm{x}$ & - & LI & FM 554 \\
\hline Temnadenia violacea (Vell.) Miers & - & $\mathrm{x}$ & $\mathrm{x}$ & - & $\mathrm{LI}$ & FM 342; RC 2120 \\
\hline \multicolumn{7}{|l|}{ ARACEAE } \\
\hline Philodendron sp. & - & - & - & $\mathrm{x}$ & $\mathrm{EP}$ & FM 1955 \\
\hline \multicolumn{7}{|l|}{ ARALIACEAE } \\
\hline Schefflera macrocarpa (Cham. \& Schltdl.) Frodin & - & $\mathrm{x}$ & $\mathrm{x}$ & - & $\mathrm{AV}$ & FM 183, 429 \\
\hline \multicolumn{7}{|l|}{ ASTERACEAE } \\
\hline Acanthospermum australe (Loefl.) Kuntze* & - & $\mathrm{x}$ & - & - & ET & FM 450 \\
\hline Achyrocline albicans Griseb. & - & $\mathrm{x}$ & - & - & $\mathrm{AB}$ & FM 870 \\
\hline Achyrocline satureioides (Lam.) DC.* & - & $\mathrm{x}$ & - & - & $\mathrm{AB}$ & FM 893 \\
\hline Ageratum conyzoides $\mathrm{L} . *$ & - & $\mathrm{x}$ & - & - & $\mathrm{AB}$ & FM 774 \\
\hline Ageratum fastigiatum (Gadner) R.M. King \& H. Rob. & - & $\mathrm{x}$ & - & - & $\mathrm{AB}$ & FM 868, 878 \\
\hline Aspilia foliacea (Spreng.) Baker & - & $\mathrm{x}$ & - & - & SB & FM 264 \\
\hline Aspilia reflexa (Baker) Baker & - & $\mathrm{x}$ & - & - & SB & FM 555 \\
\hline Ayapana amygdalina (Lam.) R.M. King \& H. Rob. & - & $\mathrm{x}$ & - & - & SB & FM 860 \\
\hline Baccharis aphylla (Vell.) DC. & - & $\mathrm{x}$ & - & - & SB & FM 761 \\
\hline Baccharis cilindrica (Less) DC. & - & $\mathrm{x}$ & - & - & SB & FM 454 \\
\hline Baccharis dracunculifollia DC. & - & $\mathrm{x}$ & - & - & SB & FM 895 \\
\hline
\end{tabular}


Tabela 1. Continuação...

\begin{tabular}{|c|c|c|c|c|c|c|}
\hline \multicolumn{7}{|c|}{ Angiospermas } \\
\hline Família/Espécie & Status & $\mathbf{C}$ & $\mathbf{R}$ & $\mathbf{M}$ & Hábito & Voucher \\
\hline Baccharis helichrysoides DC. & - & $\mathrm{x}$ & - & - & $\mathrm{AB}$ & FM 847 \\
\hline Baccharis leptocephala DC. & - & $\mathrm{x}$ & - & - & SB & FM 180, 451, 672, 861, 863 \\
\hline Baccharis serrulata (Lam.) Pers. & - & $\mathrm{x}$ & - & - & $\mathrm{AB}$ & FM 779 \\
\hline Baccharis cf. subdentata DC. & - & - & $\mathrm{x}$ & - & SB & FM 824 \\
\hline Campuloclinium megacephalum (Mart. ex Baker) R.M. King \& H. Rob. & - & $\mathrm{x}$ & - & - & SB & FM 933 \\
\hline Chresta sphaerocephala DC. & VU & $\mathrm{x}$ & $\mathrm{x}$ & - & $\mathrm{AB}$ & FM 51, 186 \\
\hline Chromolaena campestris (DC.) R.M. King & - & $\mathrm{x}$ & $\mathrm{x}$ & - & SB & FM 873 \\
\hline Chromolaena hormonioides DC. & - & $\mathrm{x}$ & $\mathrm{x}$ & - & SB & FM 655,877 \\
\hline Chromolaena oxylepis (DC.) R.M. King \& H. Rob. & - & $\mathrm{x}$ & $\mathrm{x}$ & - & SB & FM 165 \\
\hline Chromolaena pedunculosa (Hook. \& Arn.) R.M. King \& H. Rob. & - & $\mathrm{x}$ & $\mathrm{x}$ & - & SB & FM 942 \\
\hline Conoclinium macrocephalum (Less.) DC. ex B.L. Turner & - & $\mathrm{x}$ & $\mathrm{x}$ & - & SB & FM 849 \\
\hline Dasyphyllum spinescens (Less.) Cabrera & M.Rara & - & - & $\mathrm{x}$ & $\mathrm{AV}$ & FM 1167 \\
\hline Dimerostem mabrasilianum Cass. & - & $\mathrm{x}$ & - & - & SB & FM 932 \\
\hline Echinocoryne schwenkiifolia (Mart. ex DC.) H. Rob. & - & $\mathrm{x}$ & - & - & SB & FM 846 \\
\hline Elephantopus angustifolius (Sw.) Gleason & - & $\mathrm{x}$ & - & - & SB & FM 450B \\
\hline Elephantopus micropappus Less. & - & $\mathrm{x}$ & - & - & SB & FM 943 \\
\hline Elephantopus mollis Kunth* & - & $\mathrm{x}$ & - & - & SB & FM 654 \\
\hline Emilia sonchifolia (L.) DC.* & - & $\mathrm{x}$ & $\mathrm{x}$ & - & ET & FM 778 \\
\hline Eremanthus erythropappus (DC.) MacLeish & - & $\mathrm{x}$ & $\mathrm{x}$ & - & $\mathrm{AV}$ & FM 175 \\
\hline Eremanthus glomerulatus Less. & - & $\mathrm{x}$ & $\mathrm{x}$ & - & $\mathrm{AV}$ & FM 217, 656 \\
\hline Eupatorium dictyophyllum DC. & - & $\mathrm{x}$ & - & - & $\mathrm{AB}$ & FM 882 \\
\hline Gochnatia paniculata (Less.) Cabrera & Rara & $\mathrm{x}$ & - & - & $\mathrm{AB}$ & FM 210 \\
\hline Gochnatia polymorpha (Less.) Cabrera & - & $\mathrm{x}$ & - & $\mathrm{x}$ & AV & FM 748 \\
\hline Grazielia dimorpholepis (Baker) R.M. King \& H. Rob. & - & $\mathrm{x}$ & - & - & $\mathrm{AB}$ & FM 872 \\
\hline Heterocondylus alatus (Vell.) R.M. King \& H. Rob. & - & $\mathrm{x}$ & - & - & $\mathrm{AB}$ & FM 888 \\
\hline Heterocondylus amphidictyus (DC.) R.M. King \& H. Rob. & - & $\mathrm{x}$ & - & - & SB & FM 777, 899 \\
\hline Hoehnephytum trixoides (Gardner) Cabrera & - & $\mathrm{x}$ & - & - & SB & FM 224 \\
\hline Inulepsis camporum (Gardner) G.L. Neson & - & $\mathrm{x}$ & $\mathrm{x}$ & - & ET & FM 262 \\
\hline Inulopsis scaposa (Remy) O. Hoffm. & - & $\mathrm{x}$ & $\mathrm{x}$ & - & ET & FM 487 \\
\hline Koanophyllon adamantium (Gardner) R.M. King \& H. Rob. & - & $\mathrm{x}$ & $\mathrm{x}$ & - & $\mathrm{AB}$ & FM 848 \\
\hline Koanophyllon myrtilloides (DC.) R.M. King \& H. Rob. & - & $\mathrm{x}$ & $\mathrm{x}$ & - & $\mathrm{AB}$ & FM 291, 547, 762 \\
\hline Lepdaploa rufogrisea (A. St.-Hil.) H. Rob. & - & $\mathrm{x}$ & - & - & SB & FM 172 \\
\hline Lessingianthus bardanoides (Less.) H. Rob. & - & $\mathrm{x}$ & - & - & SB & FM 648 \\
\hline Lessingianthus desertorum (Mart. ex DC.) H. Rob. & - & $\mathrm{x}$ & $\mathrm{x}$ & - & ET & FM 261 \\
\hline Lessingianthus laevigatus (Mart. ex DC.) H. Rob. & - & $\mathrm{x}$ & - & - & SB & FM 865 \\
\hline Lessingianthus pycnostachyus (DC.) H. Rob. & - & $\mathrm{x}$ & - & - & SB & FM 845 \\
\hline Lessingianthus simplex (Less.) H. Rob. & - & $\mathrm{x}$ & $\mathrm{x}$ & - & ET & FM 263, 749 \\
\hline Lucilia lycopodioides (Less.) S.E. Freire & - & $\mathrm{x}$ & - & - & SB & FM 867 \\
\hline Mikania hirsutissima DC. & - & - & - & $\mathrm{x}$ & LI & FM 1185 \\
\hline Mikania oblongifolia DC. & - & $\mathrm{x}$ & $\mathrm{x}$ & - & $\mathrm{AB}$ & FM 290 \\
\hline Mikania sessilifolia DC. & - & $\mathrm{x}$ & $\mathrm{x}$ & - & $\mathrm{AB}$ & FM 170 \\
\hline Piptocarpha rotundifolia (Less.) Baker & - & $\mathrm{x}$ & - & - & $\mathrm{AV}$ & FM 658 \\
\hline Porophyllum ruderale (Jacq.) Cass.* & - & $\mathrm{x}$ & $\mathrm{x}$ & - & $\mathrm{AB}$ & FM 775 \\
\hline Praxelis kleinioides (Kunth) Sch. Bip. & - & $\mathrm{x}$ & - & - & ET & FM 665,873 \\
\hline Pseudobrickellia brasiliensis (Spreng.) R.M. King \& H. Rob. & M.Rara & - & $\mathrm{x}$ & - & $\mathrm{AV}$ & FM 866 \\
\hline Pterocaulon virgatum (L.) DC.* & - & $\mathrm{x}$ & $\mathrm{x}$ & - & SB & FM 832 \\
\hline Richterago radiata (Vell.) Roque* & - & $\mathrm{x}$ & $\mathrm{x}$ & - & ET & FM 776 \\
\hline Stevia lundiana DC. & - & $\mathrm{x}$ & - & - & SB & FM 901 \\
\hline Vernonanthura ferruginea (Less.) H. Rob. & - & $\mathrm{x}$ & - & - & $\mathrm{AV}$ & FM 748 \\
\hline Vernonia cognata Less.* & - & $\mathrm{x}$ & - & - & $\mathrm{AB}$ & FM 780 \\
\hline
\end{tabular}


Tabela 1. Continuação...

\begin{tabular}{|c|c|c|c|c|c|c|}
\hline \multicolumn{7}{|c|}{ Angiospermas } \\
\hline Família/Espécie & Status & $\mathbf{C}$ & $\mathbf{R}$ & $\mathbf{M}$ & Hábito & Voucher \\
\hline Vernonia herbacea (Vell.) Rusby & - & $\mathrm{x}$ & - & - & SB & FM 762 \\
\hline Vernonia lacunosa Mart. ex DC. & - & $\mathrm{x}$ & - & - & SB & FM 941 \\
\hline Vernonia polyanthes Less.* & - & $\mathrm{x}$ & - & - & AV & FM 457 \\
\hline Vernonia tragiaefolia DC. & - & $\mathrm{x}$ & - & - & SB & FM 874 \\
\hline Viguiera robusta Gardner & - & $\mathrm{x}$ & $\mathrm{x}$ & - & $\mathrm{AB}$ & FM 43 \\
\hline \multicolumn{7}{|l|}{ BIGNONIACEAE } \\
\hline Anemopaegma arvense (Vell.) Stellfeld \& J.F. Souza & - & $\mathrm{x}$ & $\mathrm{x}$ & - & LI & FM 295, 451 \\
\hline Anemopaegma chaimberlaynii (Sims.) Bureau \& K. Schum. & - & - & $\mathrm{x}$ & - & LI & FM 169 \\
\hline Arrabidaea brachypoda (DC.) Bureau & - & $\mathrm{x}$ & $\mathrm{x}$ & - & $\mathrm{AB}$ & FM 51 \\
\hline Cybistax antisyphilitica (Mart.) Mart. & - & $\mathrm{x}$ & $\mathrm{x}$ & - & $\mathrm{AV}$ & FM 292 \\
\hline Fridericia speciosa Mart. & - & - & - & $\mathrm{x}$ & LI & FM 1964 \\
\hline Handroanthus serratifolius (Vahl) S. Grose & - & - & - & $\mathrm{x}$ & AV & FM 1173 \\
\hline Jacaranda caroba (Vell.) DC. & - & $\mathrm{x}$ & $\mathrm{x}$ & - & $\mathrm{AB}$ & FM 293, 523 \\
\hline Jacaranda decurrens Cham. & - & $\mathrm{x}$ & $\mathrm{x}$ & - & SB & FM 293B \\
\hline Lundia sp. & - & - & - & $\mathrm{x}$ & LI & FM 1154 \\
\hline Memora pedunculata (Vell.) Miers & - & - & $\mathrm{x}$ & - & $\mathrm{AB}$ & FM 915 \\
\hline Pyrostegia venusta (Ker Gawl.) Miers* & - & $\mathrm{x}$ & $\mathrm{x}$ & - & LI & FM 920 \\
\hline Zeyheria montana Mart. & - & $\mathrm{x}$ & $\mathrm{x}$ & - & AV & FM 52 \\
\hline \multicolumn{7}{|l|}{ BORAGINACEAE } \\
\hline Cordia sellowiana Cham. & - & $\mathrm{x}$ & - & $\mathrm{x}$ & AV & FM 2168 \\
\hline Heliotropium aff. fruticosum L. & - & - & $\mathrm{x}$ & - & SB & FM 47 \\
\hline \multicolumn{7}{|l|}{ BROMELIACEAE } \\
\hline Dyckia aff. saxatilis $\mathrm{Mez}$ & - & $\mathrm{x}$ & $\mathrm{x}$ & - & ET & FM 235 \\
\hline \multicolumn{7}{|l|}{ BURSERACEAE } \\
\hline Protium widgrenii Engl. & - & $\mathrm{x}$ & $\mathrm{x}$ & $\mathrm{x}$ & $\mathrm{AV}$ & FM 476 \\
\hline \multicolumn{7}{|l|}{ CAMPANULACEAE } \\
\hline Lobelia camporum Pohl & - & $\mathrm{x}$ & $\mathrm{x}$ & - & SB & FM 782 \\
\hline Siphocampylus corymbifer Pohl & - & $\mathrm{x}$ & - & $\mathrm{x}$ & $\mathrm{AB}$ & FM 48 \\
\hline Wahlenbergia brasiliensis Cham. & - & $\mathrm{x}$ & $\mathrm{x}$ & - & SB & FM 316,863 \\
\hline \multicolumn{7}{|l|}{ CANNABACEAE } \\
\hline Celtis brasiliensis (Gardner) Planch. & - & $\mathrm{x}$ & - & - & $\mathrm{AV}$ & FM 764 \\
\hline \multicolumn{7}{|l|}{ CARYOPHYLLACEAE } \\
\hline Polycarpaea corymbosa (L.) Lam. & - & $\mathrm{x}$ & $\mathrm{x}$ & - & SB & FM 764 \\
\hline \multicolumn{7}{|l|}{ CELASTRACEAE } \\
\hline Cheiloclinium cognatum (Miers) A.C. Sm. & - & - & - & $\mathrm{x}$ & $\mathrm{AV}$ & FM 1159 \\
\hline \multicolumn{7}{|l|}{ CLUSIACEAE } \\
\hline Kielmeyera coriacea Mart. \& Zucc. & - & $\mathrm{x}$ & $\mathrm{x}$ & - & $\mathrm{AV}$ & FM 310 \\
\hline \multicolumn{7}{|l|}{ COMMELINACEAE } \\
\hline Commelina erecta $\mathrm{L}$. & - & - & $\mathrm{x}$ & - & ET & FM 484 \\
\hline Dichorisandra hexandra (Aubl.) Kuntze ex Hand.-Mazz & - & - & - & $\mathrm{x}$ & $\mathrm{EE}$ & FM 1952 \\
\hline \multicolumn{7}{|l|}{ CONVOLVULACEAE } \\
\hline Evolvulus elegans Moric. & - & $\mathrm{x}$ & - & - & LI & FM 766 \\
\hline Ipomoea argenta Meisn. & VU & - & $\mathrm{x}$ & - & SB & FM 667 \\
\hline Ipomoea nil (L.) Roth & - & $\mathrm{x}$ & $\mathrm{x}$ & - & LI & FM 769 \\
\hline Ipomoea sp.1 & - & $\mathrm{x}$ & $\mathrm{x}$ & - & LI & FM 273 \\
\hline Iротоеа sp. 2 & - & $\mathrm{x}$ & $\mathrm{x}$ & - & LI & FM 894 \\
\hline Jaquemontia sp. & - & $\mathrm{x}$ & $\mathrm{x}$ & - & LI & FM 802,854 \\
\hline
\end{tabular}


Tabela 1. Continuação...

\begin{tabular}{|c|c|c|c|c|c|c|}
\hline \multicolumn{7}{|c|}{ Angiospermas } \\
\hline Família/Espécie & Status & $\mathbf{C}$ & $\mathbf{R}$ & $\mathbf{M}$ & Hábito & Voucher \\
\hline Merremia flagellaris (Choisy) O'Donell & - & $\mathrm{x}$ & $\mathrm{x}$ & - & LI & FM 38, 670 \\
\hline Merremia tomentosa Hallierf. & - & $\mathrm{x}$ & $\mathrm{x}$ & - & SB & FM 36 \\
\hline \multicolumn{7}{|l|}{ CUCURBITACEAE } \\
\hline Cayaponia espelina (Manso) Cogn. & - & $\mathrm{x}$ & $\mathrm{x}$ & - & LI & FM 477 \\
\hline Melancium campestre Naudin & - & $\mathrm{x}$ & - & - & LI & FM 840 \\
\hline \multicolumn{7}{|l|}{ CUNONIACEAE } \\
\hline Lamanonia ternata Vell. & - & $\mathrm{x}$ & - & $\mathrm{x}$ & AV & FM 345 \\
\hline \multicolumn{7}{|l|}{ CYPERACEAE } \\
\hline Bulbostylis capillaris (L.) C.B. Clarke* & - & $\mathrm{x}$ & $\mathrm{x}$ & - & ET & FM 545 \\
\hline Bulbostylis junciformis (Kunth) C.B. Clarke & - & $\mathrm{x}$ & $\mathrm{x}$ & - & ET & FM 805 \\
\hline Bulbostylis paradoxa (Spreng.) Lindm. & - & $\mathrm{x}$ & $\mathrm{x}$ & - & ET & FM 750 \\
\hline Bulbostylis sp.1 & - & $\mathrm{x}$ & $\mathrm{x}$ & - & ET & FM 813 \\
\hline Bulbostylis sp. 2 & - & $\mathrm{x}$ & $\mathrm{x}$ & - & ET & FM 641, 804 \\
\hline Bulbostylis sp. 3 & - & $\mathrm{x}$ & $\mathrm{x}$ & - & ET & FM 862 \\
\hline Cyperus brevifolius (Rottb.) Endl. ex Hassk.* & - & $\mathrm{x}$ & $\mathrm{x}$ & - & ET & FM 806 \\
\hline Cyperus cayennensis Willd. ex Link & - & $\mathrm{x}$ & $\mathrm{x}$ & - & ET & FM 635 \\
\hline Cyperus laxus Lam. & - & $\mathrm{x}$ & $\mathrm{x}$ & - & ET & FM 817 \\
\hline Cyperus pohlii (Nees) Steud. & - & $\mathrm{x}$ & $\mathrm{x}$ & - & ET & FM 816 \\
\hline Cyperus sesquiflorus (Tor.) Mattf. \& Kük.* & - & $\mathrm{x}$ & $\mathrm{x}$ & - & ET & FM 814 \\
\hline Fimbristylis sp. & - & $\mathrm{x}$ & $\mathrm{x}$ & - & ET & FM 642, 644 \\
\hline Rhynchopora cosanguinea (Kunth) Bocck. & - & $\mathrm{x}$ & $\mathrm{x}$ & - & ET & FM 820 \\
\hline Rhynchospora exaltata Kunth & - & - & - & $\mathrm{x}$ & $\mathrm{EE}$ & FM 1176, 1182 \\
\hline Rhynchospora globosa (Kunth) Roem. \& Schult. & QA & $\mathrm{x}$ & $\mathrm{x}$ & - & ET & FM 803 \\
\hline Rhynchospora setigera Griseb. & - & $\mathrm{x}$ & $\mathrm{x}$ & - & ET & FM 359, 546, 910 \\
\hline Rhynchospora termilalis (Nees) Steud. & - & $\mathrm{x}$ & $\mathrm{x}$ & - & ET & FM 751 \\
\hline Rhynchospora sp. & - & $\mathrm{x}$ & - & - & ET & FM 806 \\
\hline Scleria bracteata Cav. & - & - & - & $\mathrm{x}$ & $\mathrm{EE}$ & FM 1166, 1170 \\
\hline \multicolumn{7}{|l|}{ DILLENIACEAE } \\
\hline Davilla rugosa Poir. & - & - & $\mathrm{x}$ & - & $\mathrm{AB}$ & FM 182 \\
\hline \multicolumn{7}{|l|}{ EBENACEAE } \\
\hline Diospyros hispida A. DC. & - & $\mathrm{x}$ & - & - & AV & FM 546 \\
\hline \multicolumn{7}{|l|}{ ERIOCAULACEAE } \\
\hline Paepalanthus cachambuensis Silveira & - & $\mathrm{x}$ & - & - & ET & FM 869, 902 \\
\hline \multicolumn{7}{|l|}{ ERYTHROXYLACEAE } \\
\hline Erythroxylum campestre A. St.-Hil. & - & $\mathrm{x}$ & $\mathrm{x}$ & - & $\mathrm{AB}$ & FM 173, 206, 275, 356 \\
\hline Erythroxylum deciduum A. St.-Hil. & - & $\mathrm{x}$ & $\mathrm{x}$ & - & $\mathrm{AB}$ & FM 277 \\
\hline Erythroxylum suberosum A. St.-Hil. & - & $\mathrm{x}$ & $\mathrm{x}$ & - & $\mathrm{AB}$ & FM 184, 303 \\
\hline Erythroxylum tortuosum Mart. & - & $\mathrm{x}$ & $\mathrm{x}$ & - & $\mathrm{AB}$ & FM 302 \\
\hline \multicolumn{7}{|l|}{ EUPHORBIACEAE } \\
\hline Alchornea sidifolia Müll. Arg. & M.Rara & - & - & $\mathrm{x}$ & $\mathrm{AV}$ & FM 1962 \\
\hline Aparisthmium cordatum (Juss.) Baill. & - & - & - & $\mathrm{x}$ & AV & FM 2088 \\
\hline Astraea lobata (L.) Klotzsch & - & - & - & $\mathrm{x}$ & $\mathrm{AB}$ & FM 2082 \\
\hline Chamaesyce caecorum (Mart. ex Boiss.) Croizat & - & $\mathrm{x}$ & $\mathrm{x}$ & - & ET & FM 200, 284 \\
\hline Croton antisyphiliticus Mart. & - & $\mathrm{x}$ & $\mathrm{x}$ & - & SB & FM 276 \\
\hline Croton glandulosus L.* & - & $\mathrm{x}$ & $\mathrm{x}$ & - & SB & FM 785 \\
\hline Croton urucurana Baill. & - & - & - & $\mathrm{x}$ & AV & FM 1153 \\
\hline Dalechampia stipulacea Müll. Arg. & - & - & - & $\mathrm{x}$ & LI & FM 475 \\
\hline Pera glabrata (Schott) Poepp. ex Baill. & - & $\mathrm{x}$ & $\mathrm{x}$ & $\mathrm{x}$ & AV & FM 479, 452, 1156; RC 2123 \\
\hline Plukenetia serrata (Vell.) L.J. Gillespie & - & - & - & $\mathrm{x}$ & LI & FM 481, 1168 \\
\hline Sebastiania bidentata (Mart. \& Zucc.) J. Paxson & - & $\mathrm{x}$ & $\mathrm{x}$ & - & SB & FM 650 \\
\hline
\end{tabular}


Tabela 1. Continuação...

\begin{tabular}{|c|c|c|c|c|c|c|}
\hline \multicolumn{7}{|c|}{ Angiospermas } \\
\hline Família/Espécie & Status & $\mathbf{C}$ & $\mathbf{R}$ & M & Hábito & Voucher \\
\hline Sebastiania commersoniana (Baill.) L.B. Sm. \& Downs & - & - & - & $\mathrm{x}$ & AV & FM 1164 \\
\hline \multicolumn{7}{|l|}{ FABACEAE } \\
\hline Aeschynomene falcata (Poir.) DC.* & - & $\mathrm{x}$ & - & - & $\mathrm{AB}$ & FM 841 \\
\hline Anadenanthera colubrina (Vell.) Brenan & - & - & - & $\mathrm{x}$ & $\mathrm{AV}$ & FM 1963 \\
\hline Andira fraxinifolia Benth. & - & $\mathrm{x}$ & $\mathrm{x}$ & $\mathrm{x}$ & $\mathrm{AV} / \mathrm{SB}$ & FM $212,343,561,1174$ \\
\hline Bauhinia forficata Link & - & - & - & $\mathrm{x}$ & AV & FM 1957 \\
\hline Bauhinia holophylla (Bong.) Steud. & - & $\mathrm{x}$ & $\mathrm{x}$ & - & $\mathrm{AB}$ & FM 212, 266 \\
\hline Chamaecrista cathartica (Mart.) H.S. Irwin \& Barneby & - & $\mathrm{x}$ & $\mathrm{x}$ & - & $\mathrm{AB}$ & FM 40; RC 2118 \\
\hline Chamaecrista rotundifolia (Pers.) Greene* & - & $\mathrm{x}$ & $\mathrm{x}$ & - & SB & FM 653, 844 \\
\hline Clitoria guianensis (Aubl.) Benth. & - & $\mathrm{x}$ & $\mathrm{x}$ & - & SB & FM 265 \\
\hline Copaifera langsdorffii Desf. & - & $\mathrm{x}$ & $\mathrm{x}$ & $\mathrm{x}$ & $\mathrm{AV}$ & FM 343 \\
\hline Crotalaria paulina Sckrank & - & $\mathrm{x}$ & - & - & SB & FM 913 \\
\hline Crotalaria retusa $\mathrm{L}$. & - & $\mathrm{x}$ & - & - & SB & FM 822 \\
\hline Crotalaria sp. & - & $\mathrm{x}$ & - & - & SB & FM 861 \\
\hline Dalbergia glaucescens (Mart. ex Benth.) Benth. & Rss. & $\mathrm{x}$ & $\mathrm{x}$ & - & $\mathrm{AV}$ & FM 429, 646 \\
\hline Desmodium incanum DC. & - & $\mathrm{x}$ & $\mathrm{x}$ & - & ET & FM 473, 748 \\
\hline Enterolobium gummiferum (Mart.) J.F. Macbr. & - & $\mathrm{x}$ & - & - & $\mathrm{AV}$ & FM 280 \\
\hline Eriosema heterophyllum Benth. & - & $\mathrm{x}$ & - & - & SB & FM 472, 1179 \\
\hline Galactia boavista (Vell.) Burkart & - & $\mathrm{x}$ & $\mathrm{x}$ & - & SB & FM 283 \\
\hline Hymenaea courbaril $\mathrm{L}$. & - & - & - & $\mathrm{x}$ & $\mathrm{AV}$ & FM 474 \\
\hline Indigofera suffruticosa Mill.* & - & $\mathrm{x}$ & - & - & $\mathrm{AB}$ & FM 853 \\
\hline Leptolobium elegans Vogel & - & $\mathrm{x}$ & $\mathrm{x}$ & - & $\mathrm{AV}$ & FM 483, 562 \\
\hline Leucochloron incuriale (Vell.) Barneby \& J.W. Grimes & - & $\mathrm{x}$ & - & $\mathrm{x}$ & $\mathrm{AV}$ & FM 1958 \\
\hline Machaerium nictitans (Vell.) Benth. & - & - & - & $\mathrm{x}$ & $\mathrm{AV}$ & FM 1951 \\
\hline Machaerium villosum Vogel & - & - & - & $\mathrm{x}$ & $\mathrm{AV}$ & FM 1160 \\
\hline Ormosia arborea (Vell.) Harms & - & - & - & $\mathrm{x}$ & $\mathrm{AV}$ & FM 1175 \\
\hline Periandra mediterranea (Vell.) Taub. & - & $\mathrm{x}$ & $\mathrm{x}$ & - & SB & FM 45 \\
\hline Platycyamus regnellii Benth. & - & - & - & $\mathrm{x}$ & AV & FM 1947 \\
\hline Senna rugosa (G. Don.) H.S. Irwin \& Barneby & - & $\mathrm{x}$ & $\mathrm{x}$ & - & $\mathrm{AV}$ & FM 42B; RC 2119 \\
\hline Stylosanthes grandifolia Brandão \& Sousa Costa & - & $\mathrm{x}$ & $\mathrm{x}$ & - & SB & FM 834 \\
\hline Stylosanthes guianensis (Aubl.) Sw.* & - & $\mathrm{x}$ & $\mathrm{x}$ & - & SB & FM 793 \\
\hline Stylosanthes scabra Vogel & - & $\mathrm{x}$ & $\mathrm{x}$ & - & SB & FM 792 \\
\hline Stylosanthes sp. & - & $\mathrm{x}$ & $\mathrm{x}$ & - & SB & FM 755 \\
\hline Zornia reticulata $\mathrm{Sm}$. & - & $\mathrm{x}$ & $\mathrm{x}$ & - & SB & FM 892 \\
\hline \multicolumn{7}{|l|}{ GENTIANACEAE } \\
\hline Calolisianthus speciosus (Cham. \& Schltdl.) Gilg & - & $\mathrm{x}$ & $\mathrm{x}$ & - & ET & FM 42 \\
\hline Curtia patula Knobl. & - & - & $\mathrm{x}$ & - & ET & FM 668 \\
\hline Deianira chiquitana Herzog & - & $\mathrm{x}$ & $\mathrm{x}$ & - & ET & FM 41 \\
\hline \multicolumn{7}{|l|}{ GESNERIACEAE } \\
\hline Sinningia allagophylla (Mart.) Wiehler & - & $\mathrm{x}$ & - & - & ET & FM 940 \\
\hline \multicolumn{7}{|l|}{ HYPERICACEAE } \\
\hline Hypericum brasiliense Choisy & - & $\mathrm{x}$ & - & - & ET & FM 2086 \\
\hline \multicolumn{7}{|l|}{ IRIDACEAE } \\
\hline Sisyrinchium sp. & - & $\mathrm{x}$ & $\mathrm{x}$ & - & ET & FM 830 \\
\hline Trimezia juncifolia Klatt & - & $\mathrm{x}$ & $\mathrm{x}$ & - & ET & FM 309 \\
\hline \multicolumn{7}{|l|}{ LAMIACEAE } \\
\hline Aegiphila lhotskiana Cham. & - & $\mathrm{x}$ & $\mathrm{x}$ & - & $\mathrm{AV}$ & FM 453 \\
\hline Clinopodium repens (D. Don) Benth. & - & $\mathrm{x}$ & $\mathrm{x}$ & - & SB & FM 664 \\
\hline Hyptidendron canum (Pohl) Harley & Rara & $\mathrm{x}$ & $\mathrm{x}$ & - & $\mathrm{AV}$ & FM 208, 862, 875, 876 \\
\hline Hyptis amaroucaulos Brig. & - & $\mathrm{x}$ & $\mathrm{x}$ & - & $\mathrm{AB}$ & FM 896 \\
\hline
\end{tabular}


Tabela 1. Continuação...

\begin{tabular}{|c|c|c|c|c|c|c|}
\hline \multicolumn{7}{|c|}{ Angiospermas } \\
\hline Família/Espécie & Status & $\mathbf{C}$ & $\mathbf{R}$ & M & Hábito & Voucher \\
\hline Hyptis crinita Benth. & - & $\mathrm{x}$ & $\mathrm{x}$ & - & $\mathrm{AB}$ & FM 171, 459 \\
\hline Hyptis marrubioides Epling & - & $\mathrm{x}$ & $\mathrm{x}$ & - & $\mathrm{AB}$ & FM 666, 846 \\
\hline Hyptis plectranthoides Benth. & - & $\mathrm{x}$ & $\mathrm{x}$ & - & $\mathrm{AB}$ & FM 794 \\
\hline Hyptis rugosa Benth. & - & $\mathrm{x}$ & $\mathrm{x}$ & - & $\mathrm{AB}$ & FM 864 \\
\hline Hyptis sp. & - & $\mathrm{x}$ & $\mathrm{x}$ & - & $\mathrm{AB}$ & FM 225, 226 \\
\hline Hyptis virgata Benth. & - & $\mathrm{x}$ & $\mathrm{x}$ & - & SB & FM 269, 282, 1183 \\
\hline Rhabdocaulon denudatum (Benth.) Epling & - & $\mathrm{x}$ & - & - & SB & FM 171B \\
\hline Vitex polygama Cham. & - & $\mathrm{x}$ & $\mathrm{x}$ & - & AV & FM 346 \\
\hline \multicolumn{7}{|l|}{ LAURACEAE } \\
\hline Nectandra lanceolata Nees & - & - & - & $\mathrm{x}$ & AV & FM 485 \\
\hline Nectandra nitidula Nees & - & - & - & $\mathrm{x}$ & AV & FM 1157 \\
\hline Ocotea odorifera Mart. & VU & - & - & $\mathrm{x}$ & AV & FM 850 \\
\hline Persea major (Nees) L.E. Kopp & Rara & - & $\mathrm{x}$ & - & AV & RC 2127 \\
\hline \multicolumn{7}{|l|}{ LECYTHIDACEAE } \\
\hline Cariniana estrellensis (Raddi) Kuntze & - & - & - & $\mathrm{x}$ & AV & FM 921 \\
\hline \multicolumn{7}{|l|}{ LYTHRACEAE } \\
\hline Cuphea carthagenensis (Jacq.) J.F. Macbr. & - & $\mathrm{x}$ & $\mathrm{x}$ & - & ET & FM 831 \\
\hline Cuphea mesostemon Koehne & - & $\mathrm{x}$ & $\mathrm{x}$ & - & ET & FM 827 \\
\hline Cuphea thymoides Cham. \& Schltdl. & - & $\mathrm{x}$ & $\mathrm{x}$ & - & ET & FM 274, 486 \\
\hline Diplusodon virgatus Pohl & - & $\mathrm{x}$ & $\mathrm{x}$ & - & $\mathrm{AB}$ & FM 45, 343 \\
\hline \multicolumn{7}{|l|}{ MALPIGHIACEAE } \\
\hline Banisteriopsis argyrophylla (A. Juss.) B. Gates & - & $\mathrm{x}$ & $\mathrm{x}$ & - & $\mathrm{AB}$ & RC 2130 \\
\hline Banisteriopsis campestris (A. Juss.) Little & - & $\mathrm{x}$ & $\mathrm{x}$ & - & $\mathrm{AB}$ & FM 30, 258 \\
\hline Byrsonima coccolobifolia Kunth & - & $\mathrm{x}$ & $\mathrm{x}$ & - & AV & FM 259, 304 \\
\hline Byrsonima intermedia A. Juss. & - & $\mathrm{x}$ & $\mathrm{x}$ & - & $\mathrm{AB}$ & FM 460, 548; RC 2125 \\
\hline Byrsonima laxiflora Griseb. & - & - & - & $\mathrm{x}$ & AV & FM 1155 \\
\hline Byrsonima verbascifolia (L.) DC. & - & $\mathrm{x}$ & - & - & AV & FM 549 \\
\hline Camarea affinis A. St.-Hil. & - & $\mathrm{x}$ & - & - & ET & FM 784, 821 \\
\hline Camarea ericoides A. St.-Hil. & - & $\mathrm{x}$ & - & - & ET & FM 231, 289 \\
\hline Camarea hyrsuta A. St.-Hil. & VU & $\mathrm{x}$ & - & - & ET & FM 767 \\
\hline Heteropterys umbellata A. Juss. & - & $\mathrm{x}$ & $\mathrm{x}$ & - & $\mathrm{AB}$ & FM 260, 288, 550 \\
\hline Peixotoa tomentosa A. Juss. & - & $\mathrm{x}$ & - & - & LI & FM 50, 287 \\
\hline \multicolumn{7}{|l|}{ MALVACEAE } \\
\hline Krapovickasia macrodon (DC.) Fryxell & - & $\mathrm{x}$ & - & - & SB & FM 1959 \\
\hline Luehea grandiflora Mart. \& Zucc. & - & - & - & $\mathrm{x}$ & AV & FM 1953 \\
\hline Pavonia sp. & - & $\mathrm{x}$ & - & - & SB & FM 855 \\
\hline Peltaea edouardii (Hochr.) Krapov. \& Cristóbal & - & $\mathrm{x}$ & $\mathrm{x}$ & - & SB & FM 272, 285 \\
\hline Peltea sp. & - & $\mathrm{x}$ & $\mathrm{x}$ & - & SB & FM 860 \\
\hline Sida cordifolia L.* & - & $\mathrm{x}$ & $\mathrm{x}$ & - & SB & FM 842 \\
\hline Sida glaziovii K. Schum.* & - & $\mathrm{x}$ & $\mathrm{x}$ & - & SB & FM 343 \\
\hline Sida linifolia Cav.* & - & $\mathrm{x}$ & $\mathrm{x}$ & - & SB & FM 647 \\
\hline Sida rhombifolia L.* & - & $\mathrm{x}$ & $\mathrm{x}$ & - & SB & FM 795, 839 \\
\hline Triunfetta bartramii L.* & - & $\mathrm{x}$ & - & $\mathrm{x}$ & $\mathrm{AB}$ & FM 1948 \\
\hline Waltheria cf. communis A. St.-Hil & - & $\mathrm{x}$ & - & - & SB & FM 855 \\
\hline Waltheria douradinha A. St.-Hil & - & $\mathrm{x}$ & - & - & SB & FM 878 \\
\hline Waltheria indica $\mathrm{L} . *$ & - & $\mathrm{x}$ & $\mathrm{x}$ & - & SB & FM 453, 659 \\
\hline \multicolumn{7}{|l|}{ MELASTOMATACEAE } \\
\hline Cambessedesia espora DC. & - & $\mathrm{x}$ & $\mathrm{x}$ & - & SB & FM 227 \\
\hline Chaetostoma inerme Naudin & $\mathrm{DD}$ & $\mathrm{x}$ & $\mathrm{x}$ & - & SB & FM 787, 856 \\
\hline Leandra aurea (Cham.) Cogn. & Rara & $\mathrm{x}$ & $\mathrm{x}$ & - & AV & FM 228, 301 \\
\hline
\end{tabular}


Tabela 1. Continuação...

\begin{tabular}{|c|c|c|c|c|c|c|}
\hline \multicolumn{7}{|c|}{ Angiospermas } \\
\hline Família/Espécie & Status & $\mathbf{C}$ & $\mathbf{R}$ & M & Hábito & Voucher \\
\hline Leandra xanthocoma (Naudin) Cogn. & - & - & - & $\mathrm{x}$ & SB & FM 2084 \\
\hline Miconia albicans (Sw.) Triana & - & $\mathrm{x}$ & $\mathrm{x}$ & - & AV & FM 228, 268 \\
\hline Miconia ligustroides (DC.) Naudin & - & $\mathrm{x}$ & $\mathrm{x}$ & - & AV & FM 205, 343; RC 2122 \\
\hline Miconia rubiginosa (Bompl.) DC. & - & $\mathrm{x}$ & $\mathrm{x}$ & - & AV & FM 211, 228, 358 \\
\hline Miconia stenostachya DC. & - & $\mathrm{x}$ & $\mathrm{x}$ & - & AV & FM 267, 300 \\
\hline Microlicia fulva (Spreng.) Cham. & - & $\mathrm{x}$ & $\mathrm{x}$ & - & SB & FM 429 \\
\hline Microlicia isophylla DC. & - & $\mathrm{x}$ & $\mathrm{x}$ & - & SB & FM 790 \\
\hline Ossaea congestiflora (Naudin) Cogn. & - & $\mathrm{x}$ & $\mathrm{x}$ & - & SB & FM 791 \\
\hline Tibouchina gracilis Cogn. & - & $\mathrm{x}$ & $\mathrm{x}$ & - & SB & FM 662, 788, 843, 897 \\
\hline Tibouchina martialis (Cham.) Cogn. & - & $\mathrm{x}$ & $\mathrm{x}$ & - & $\mathrm{AB}$ & RC 2117 \\
\hline Tibouchina stenocarpa (DC.) Cogn. & - & $\mathrm{x}$ & - & $\mathrm{x}$ & AV & FM 207, 1161 \\
\hline Trembleya parviflora (D.Don.) Cogn. & - & $\mathrm{x}$ & $\mathrm{x}$ & - & AV & FM 857 \\
\hline Trembleya phlogiformis DC. & - & $\mathrm{x}$ & $\mathrm{x}$ & - & SB & FM 429, 663, 789 \\
\hline \multicolumn{7}{|l|}{ MELIACEAE } \\
\hline Cedrela fissilis Vell. & - & $\mathrm{x}$ & - & $\mathrm{x}$ & $\mathrm{AV}$ & FM 922 \\
\hline \multicolumn{7}{|l|}{ MENISPERMACEAE } \\
\hline Cissampelus ovalifolia DC. & - & $\mathrm{x}$ & $\mathrm{x}$ & - & SB & FM 347, 560 \\
\hline \multicolumn{7}{|l|}{ MORACEAE } \\
\hline Dorstenia tubicina Ruiz \& Pav. & - & $\mathrm{x}$ & $\mathrm{x}$ & - & ET & FM 480 \\
\hline Ficus pertusa L. f. & - & - & $\mathrm{x}$ & - & $\mathrm{AB}$ & FM 858 \\
\hline \multicolumn{7}{|l|}{ MYRSINACEAE } \\
\hline Myrsine guianensis (Aubl.) Kuntze & - & $\mathrm{x}$ & $\mathrm{x}$ & - & $\mathrm{AV}$ & FM 160, 178, 525 \\
\hline \multicolumn{7}{|l|}{ MYRTACEAE } \\
\hline Calyptranthes clusiifolia O. Berg & - & - & - & $\mathrm{x}$ & $\mathrm{AV}$ & FM 1950 \\
\hline Campomanesia adamantium (Cambess.) O. Berg & - & $\mathrm{x}$ & $\mathrm{x}$ & - & $\mathrm{AB}$ & FM 480B \\
\hline Campomanesia pubescens (DC.) O. Berg & - & $\mathrm{x}$ & $\mathrm{x}$ & - & $\mathrm{AB}$ & FM 205, 525, 885 \\
\hline Eugenia bimarginata DC. & - & - & $\mathrm{x}$ & - & SB & FM 675,887 \\
\hline Eugenia punicifolia (Kunth) DC. & - & $\mathrm{x}$ & $\mathrm{x}$ & - & AV & FM 296, 426, 429; RC 2116 \\
\hline Myrcia guianensis (Aubl.) DC. & - & $\mathrm{x}$ & $\mathrm{x}$ & - & AV & FM 297, 352, 426 \\
\hline Myrcia splendens (Sw.) DC. & - & $\mathrm{x}$ & $\mathrm{x}$ & $\mathrm{x}$ & AV & FM 347 \\
\hline Myrcia tomentosa (Aubl.) DC. & - & $\mathrm{x}$ & $\mathrm{x}$ & - & AV & FM 298, 552 \\
\hline Psidium cinereum Mart. ex DC. & - & $\mathrm{x}$ & $\mathrm{x}$ & - & $\mathrm{AB}$ & FM 299, 351 \\
\hline Psidium guineense $\mathrm{Sw}$. & - & $\mathrm{x}$ & $\mathrm{x}$ & - & AV & FM 352 \\
\hline Syzygium jambos (L.) Alston & - & - & - & $\mathrm{x}$ & AV & FM 464 \\
\hline \multicolumn{7}{|l|}{ NYCTAGINACEAE } \\
\hline Guapira opposita (Vell.) Reitz & - & & - & $\mathrm{x}$ & $\mathrm{AV}$ & FM 1158 \\
\hline \multicolumn{7}{|l|}{ ORCHIDACEAE } \\
\hline Cleistes rosea Lindl. & - & $\mathrm{x}$ & - & - & ET & FM 1923 \\
\hline Galeandra montana Barb. Rodr. & - & $\mathrm{x}$ & $\mathrm{x}$ & - & ET & FM 426 \\
\hline Habenaria longipedicellata Hoehne & - & $\mathrm{x}$ & - & - & ET & FM 786 \\
\hline Pelexia cf. aphylla Ridl. & - & - & $\mathrm{x}$ & - & ET & FM 876 \\
\hline Polystachya estrellensis Rchb. f. & - & $\mathrm{x}$ & - & $\mathrm{x}$ & EP & FM 836 \\
\hline \multicolumn{7}{|l|}{ OROBANCHACEAE } \\
\hline Buchnera lavandulacea Cham. \& Schultdl. & - & $\mathrm{x}$ & $\mathrm{x}$ & - & SB & FM 667 \\
\hline Esterhazya splendida J.C. Mikan & - & $\mathrm{x}$ & $\mathrm{x}$ & - & $\mathrm{AB}$ & FM 423, 671 \\
\hline \multicolumn{7}{|l|}{ PASSIFLORACEAE } \\
\hline Passiflora suberosa L. & - & - & $\mathrm{x}$ & - & LI & FM 753 \\
\hline Passiflora vellozii Gardner & - & - & $\mathrm{x}$ & - & LI & FM 917 \\
\hline \multicolumn{7}{|l|}{ PHYLLANTHACEAE } \\
\hline Phyllanthus niuri L.* & - & $\mathrm{x}$ & - & - & SB & FM 826 \\
\hline
\end{tabular}


Tabela 1. Continuação...

\begin{tabular}{|c|c|c|c|c|c|c|}
\hline \multicolumn{7}{|c|}{ Angiospermas } \\
\hline Família/Espécie & Status & $\mathbf{C}$ & $\mathbf{R}$ & $\mathbf{M}$ & Hábito & Voucher \\
\hline Phyllanthus rosellus Müll. Arg. & - & - & $\mathrm{x}$ & - & ER & FM 2090 \\
\hline \multicolumn{7}{|l|}{ PIPERACEAE } \\
\hline Peperomia oreophila Henschen & - & - & $\mathrm{x}$ & - & ER & FM 166 \\
\hline Piper gaudichaudianum Kunth & - & - & - & $\mathrm{x}$ & $\mathrm{AB}$ & FM 478 \\
\hline \multicolumn{7}{|l|}{ POACEAE } \\
\hline Andropogon bicornis L.* & - & $\mathrm{x}$ & $\mathrm{x}$ & - & ET & FM 908 \\
\hline Andropogon leucostachyus Kunth* & - & $\mathrm{x}$ & $\mathrm{x}$ & - & ET & FM 543 \\
\hline Andropogon selloanus (Hack.) Hack. & - & $\mathrm{x}$ & $\mathrm{x}$ & - & ET & FM 525 \\
\hline Anthaenantia lanata (Kunth) Benth. & - & $\mathrm{x}$ & - & - & ET & FM 872 \\
\hline Aristida ekmaniana Henrard & - & $\mathrm{x}$ & - & - & ET & FM 866 \\
\hline Aristida jubata (Arechav.) Herter & - & $\mathrm{x}$ & - & - & ET & FM 467 \\
\hline Aristida recurvata Kunth & - & $\mathrm{x}$ & $\mathrm{x}$ & - & ET & FM 624, 629 \\
\hline Axonopus aureus P. Beauv. & DD & $\mathrm{x}$ & $\mathrm{x}$ & - & ET & FM 430, 458 \\
\hline Axonopus brasiliensis (Spreng.) Kuhlm. & DD & $\mathrm{x}$ & $\mathrm{x}$ & - & ET & FM 468, 544 \\
\hline Axonopus cf. fissifolius (Raddi) Kuhlm. & - & $\mathrm{x}$ & $\mathrm{x}$ & - & ET & FM 818 \\
\hline Axonopus pressus (Nees ex Steud.) Parodi & - & $\mathrm{x}$ & $\mathrm{x}$ & - & ET & FM 626 \\
\hline Axonopus siccus (Nees) Kuhlm. & - & $\mathrm{x}$ & $\mathrm{x}$ & - & ET & FM 799 \\
\hline Chusquea tenuiglumis Döll & DD & - & - & $\mathrm{x}$ & $\mathrm{EE}$ & FM 1946 \\
\hline Ctenium cirrhosum (Ness) Kunth & - & $\mathrm{x}$ & $\mathrm{x}$ & - & ET & FM 447 \\
\hline Digitaria insularis (L.) Fedde* & - & $\mathrm{x}$ & $\mathrm{x}$ & - & ET & FM 799 \\
\hline Echinolaena inflexa (Poir.) Chase & - & $\mathrm{x}$ & $\mathrm{x}$ & - & ET & FM 176, 417 \\
\hline Eleusine indica $\mathrm{L} . *$ & - & $\mathrm{x}$ & $\mathrm{x}$ & - & ET & FM 870 \\
\hline Elionurus muticus (Spreng.) Kuntze & - & $\mathrm{x}$ & - & - & ET & FM 883 \\
\hline Eragrostis polytricha Nees & - & $\mathrm{x}$ & $\mathrm{x}$ & - & ET & FM 469, 540, 669 \\
\hline Eragrostis rufescens Schrad. ex Schult. & - & $\mathrm{x}$ & $\mathrm{x}$ & - & ET & FM 632 \\
\hline Eragrostis solida Nees & - & $\mathrm{x}$ & $\mathrm{x}$ & - & ET & FM 633 \\
\hline Eragrostis tenuifolia (A. Rich.) Hochst. ex Steud.* & - & $\mathrm{x}$ & $\mathrm{x}$ & - & ET & FM 869 \\
\hline Homolepis sp. & - & - & - & $\mathrm{x}$ & ET & FM 1954 \\
\hline Hyparrhenia rufa (Nees) Stapf* & - & - & $\mathrm{x}$ & - & ET & FM 1961 \\
\hline Ichnanthus procurrens (Ness ex Trin.) Swallen & - & $\mathrm{x}$ & - & - & ET & FM 808 \\
\hline Loudetiopsis chrysothrix (Nees) Conert & - & $\mathrm{x}$ & $\mathrm{x}$ & - & ET & FM 538 \\
\hline Melinis minutiflora $\mathrm{P}$. Beauv.* & - & $\mathrm{x}$ & $\mathrm{x}$ & - & ET & FM 923 \\
\hline Melinis repens (Willd.) Zizka* & - & $\mathrm{x}$ & $\mathrm{x}$ & - & ET & FM 541 \\
\hline Olyra humilis Nees & - & - & $\mathrm{x}$ & $\mathrm{x}$ & ET & FM 815 \\
\hline Panicum campestre Nees ex Trin. & - & $\mathrm{x}$ & $\mathrm{x}$ & - & ET & FM 909 \\
\hline Panicum cyanescens Nees ex Trin. & - & $\mathrm{x}$ & $\mathrm{x}$ & - & ET & FM 876 \\
\hline Panicum olyroides Kunth & - & $\mathrm{x}$ & $\mathrm{x}$ & - & ET & FM 797 \\
\hline Panicum pilosum $\mathrm{Sw}$. & - & - & - & $\mathrm{x}$ & ET & FM 851, 1171 \\
\hline Panicum sellowii Nees & - & - & - & $\mathrm{x}$ & ET & FM 813 \\
\hline Parodiophyllochloa pantricha (Hack.) Zuloaga \& Morrone & - & - & - & $\mathrm{x}$ & ET & FM 1960 \\
\hline Paspalum carinatum Humb. \& Bonpl. ex Flüggé & - & - & $\mathrm{x}$ & - & ET & FM 542 \\
\hline Paspalum erianthum Nees ex Trin. & - & $\mathrm{x}$ & $\mathrm{x}$ & - & ET & FM 879 \\
\hline Paspalum eucomum Nees ex Trin. & - & $\mathrm{x}$ & $\mathrm{x}$ & - & ET & FM 448 \\
\hline Paspalum gardnerianum Nees & - & $\mathrm{x}$ & $\mathrm{x}$ & - & ET & FM 538, 809 \\
\hline Paspalum geminiflorum Steud. & - & $\mathrm{x}$ & $\mathrm{x}$ & - & ET & FM 898 \\
\hline Paspalum hyalinum Nees ex Trin. & - & $\mathrm{x}$ & $\mathrm{x}$ & - & ET & FM 624, 630 \\
\hline Paspalum notatum Flüggé* & - & $\mathrm{x}$ & $\mathrm{x}$ & - & ET & FM 637 \\
\hline Paspalum polyphyllum Nees & - & $\mathrm{x}$ & $\mathrm{x}$ & - & ET & FM 639 \\
\hline Schizachyrium condensatum (Kunth) Nees & - & $\mathrm{x}$ & $\mathrm{x}$ & - & ET & FM 629 \\
\hline Schizachyrium sanguineum (Retz.) Alston & - & $\mathrm{x}$ & $\mathrm{x}$ & - & ET & FM 643 \\
\hline
\end{tabular}


Tabela 1. Continuação...

\begin{tabular}{|c|c|c|c|c|c|c|}
\hline \multicolumn{7}{|c|}{ Angiospermas } \\
\hline Família/Espécie & Status & $\mathbf{C}$ & $\mathbf{R}$ & $\mathbf{M}$ & Hábito & Voucher \\
\hline Schizachyrium tenerum Nees & - & $\mathrm{x}$ & $\mathrm{x}$ & - & ET & FM 628, 631 \\
\hline Setaria parviflora (Poir.) Kerguélen* & - & $\mathrm{x}$ & $\mathrm{x}$ & - & ET & FM 809 \\
\hline Sporobolus indicus (L.) R. Br.* & - & $\mathrm{x}$ & $\mathrm{x}$ & - & ET & FM 800 \\
\hline Sporobolus pyramidalis $\mathrm{P}$. Beauv. & - & $\mathrm{x}$ & $\mathrm{x}$ & - & ET & FM 819 \\
\hline Trachypogon spicatus (L. f.) Kuntze & - & $\mathrm{x}$ & $\mathrm{x}$ & - & ET & FM 625, 645 \\
\hline Tristachya leiostachya Nees & - & $\mathrm{x}$ & $\mathrm{x}$ & - & ET & FM 797 \\
\hline Urochloa brizantha (Hochst. ex A. Rich.) R.D. Webster* & - & $\mathrm{x}$ & $\mathrm{x}$ & - & ET & FM 796 \\
\hline \multicolumn{7}{|l|}{ POLYGALACEAE } \\
\hline Bredemeyera floribunda Willd. & - & $\mathrm{x}$ & - & - & AV & FM 832 \\
\hline Bredemeyera kunthiana Klotzsch & - & - & $\mathrm{x}$ & - & $\mathrm{AB}$ & FM 832, 914 \\
\hline Polygala galioides Poir. & - & $\mathrm{x}$ & $\mathrm{x}$ & - & ET & FM 674B \\
\hline Polygala longicaulis Kunth & - & $\mathrm{x}$ & $\mathrm{x}$ & - & ET & FM 455 \\
\hline Polygala paniculata L.* & - & $\mathrm{x}$ & $\mathrm{x}$ & - & ET & FM 674 \\
\hline Polygala urbani Chodat* & - & $\mathrm{x}$ & - & - & ET & FM 482 \\
\hline \multicolumn{7}{|l|}{ PROTEACEAE } \\
\hline Roupala montana Aubl. & - & $\mathrm{x}$ & - & - & AV & FM 867 \\
\hline \multicolumn{7}{|l|}{ ROSACEAE } \\
\hline Rubus urticifolius Poir.* & - & $\mathrm{x}$ & - & - & $\mathrm{AB}$ & FM 754, 807 \\
\hline \multicolumn{7}{|l|}{ RUBIACEAE } \\
\hline Coccocypselum lanceolatum (Ruiz \& Pav.) Pers. & - & - & $\mathrm{x}$ & $\mathrm{x}$ & SB & FM 768 \\
\hline Decleuxia diantheroides Standl. & - & $\mathrm{x}$ & $\mathrm{x}$ & - & SB & FM 179, 756 \\
\hline Declieuxia cf. fruticosa (Will. ex Roem. \& Schult.) Kuntze & - & $\mathrm{x}$ & $\mathrm{x}$ & - & SB & FM 905 \\
\hline Declieuxia cordigera Mart. \& Zucc. ex Schult. \& Schult. f. & - & $\mathrm{x}$ & $\mathrm{x}$ & - & SB & FM 44, 270, 471 \\
\hline Diodella teres (Walter) Small* & - & $\mathrm{x}$ & $\mathrm{x}$ & - & SB & FM 652 \\
\hline Faramea nigrescens Mart. & - & - & - & $\mathrm{x}$ & AV & FM 1172 \\
\hline Galianthe liliifolia (Standl.) E.L. Cabral & - & $\mathrm{x}$ & $\mathrm{x}$ & - & $\mathrm{AB}$ & FM 49, 428 \\
\hline Mitracarpus hirtus (L.) DC.* & - & $\mathrm{x}$ & $\mathrm{x}$ & - & SB & FM 556, 651 \\
\hline Palicourea rigida Kunth & - & $\mathrm{x}$ & $\mathrm{x}$ & - & $\mathrm{AB}$ & FM 53 \\
\hline Psychothria hastisepala Müll. Arg. & Rara & - & - & $\mathrm{x}$ & AV & FM 1956 \\
\hline Sabicea brasiliensis Wernham & - & $\mathrm{x}$ & $\mathrm{x}$ & - & $\mathrm{AB}$ & FM 431; RC 2126 \\
\hline Spermacoce alata Aubl.* & - & $\mathrm{x}$ & $\mathrm{x}$ & - & SB & FM 865 \\
\hline Spermacoce latifolia Aubl.* & - & $\mathrm{x}$ & $\mathrm{x}$ & - & SB & FM 174 \\
\hline Spermacoce tenella Kunth* & - & $\mathrm{x}$ & $\mathrm{x}$ & - & SB & FM 271 \\
\hline Spermacoce verticilata L.* & - & $\mathrm{x}$ & $\mathrm{x}$ & - & SB & FM 482 \\
\hline Tocoyena formosa (Cham. \& Schultdl.) K. Schum. & - & $\mathrm{x}$ & $\mathrm{x}$ & - & AV & FM 229, 312, 557; RC 2124 \\
\hline \multicolumn{7}{|l|}{ RUTACEAE } \\
\hline Dictyoloma vandellianum A. Juss. & - & - & - & $\mathrm{x}$ & AV & FM 1162 \\
\hline \multicolumn{7}{|l|}{ SALICACEAE } \\
\hline Casearia decandra Jacq. & - & - & $\mathrm{x}$ & - & AV & FM 758 \\
\hline Casearia lasiophylla Eicher & - & - & - & $\mathrm{x}$ & AV & FM 1184 \\
\hline Casearia sylvestris $\mathrm{Sw}$. & - & - & $\mathrm{x}$ & $\mathrm{x}$ & AV/SB & FM 185, 204 \\
\hline \multicolumn{7}{|l|}{ SAPINDACEAE } \\
\hline Cupania vernalis Cambess. & - & - & - & $\mathrm{x}$ & AV & FM 1949 \\
\hline Serjania erecta Radlk. & - & $\mathrm{x}$ & - & - & $\mathrm{AB}$ & FM 875 \\
\hline \multicolumn{7}{|l|}{ SIPARUNACEAE } \\
\hline Siparuna cujabana (Mart.) A. DC. & - & - & - & $\mathrm{x}$ & AV & FM 1165 \\
\hline \multicolumn{7}{|l|}{ SMILACACEAE } \\
\hline Smilax elastica Griseb. & - & $\mathrm{x}$ & $\mathrm{x}$ & - & LI & FM 201 \\
\hline
\end{tabular}


Tabela 1. Continuação...

\begin{tabular}{|c|c|c|c|c|c|c|}
\hline \multicolumn{7}{|c|}{ Angiospermas } \\
\hline Família/Espécie & Status & $\mathbf{C}$ & $\mathbf{R}$ & $\mathbf{M}$ & Hábito & Voucher \\
\hline Smilax fluminensis Steud. & - & $\mathrm{x}$ & $\mathrm{x}$ & - & LI & FM 311 \\
\hline \multicolumn{7}{|l|}{ SOLANACEAE } \\
\hline Schwenckia brasiliensis Poir.* & - & $\mathrm{x}$ & - & - & SB & FM 868 \\
\hline Solanum americanum Mill.* & - & $\mathrm{x}$ & - & - & SB & FM 924 \\
\hline Solanum capsicoides All.* & - & $\mathrm{x}$ & - & - & $\mathrm{AB}$ & FM 657 \\
\hline Solanum lycocarpum A. St.-Hil.* & - & $\mathrm{x}$ & - & - & AV & FM 833 \\
\hline \multicolumn{7}{|l|}{ STYRACACEAE } \\
\hline Styrax ferrugineus Nees \& Mart. & - & $\mathrm{x}$ & $\mathrm{x}$ & - & $\mathrm{AV}$ & FM 167, 203 \\
\hline \multicolumn{7}{|l|}{ TURNERACEAE } \\
\hline Piriqueta cistoides (L.) Griseb. & - & $\mathrm{x}$ & $\mathrm{x}$ & - & SB & FM 765 \\
\hline \multicolumn{7}{|l|}{ URTICACEAE } \\
\hline Cecropia pachystachya Trécul & - & - & - & $\mathrm{x}$ & AV & FM 2041 \\
\hline \multicolumn{7}{|l|}{ VELLOZIACEAE } \\
\hline Barbacenia tomentosa Mart. & - & - & $\mathrm{x}$ & - & ER & $\mathrm{RC} 2121$ \\
\hline \multicolumn{7}{|l|}{ VERBENACEAE } \\
\hline Lantana camara L.* & - & $\mathrm{x}$ & - & - & $\mathrm{AB}$ & FM 783 \\
\hline Lantana fucata Lindl.* & - & $\mathrm{x}$ & - & - & $\mathrm{AB}$ & FM 825 \\
\hline Lippia lupulina Cham. & - & $\mathrm{x}$ & $\mathrm{x}$ & - & $\mathrm{AB}$ & FM 302 \\
\hline Lippia rotundifolia Cham. & - & $\mathrm{x}$ & $\mathrm{x}$ & - & $\mathrm{AB}$ & FM 862 \\
\hline Stachytarpheta cayennensis (Rich.) Vahl & - & $\mathrm{x}$ & - & - & SB & FM 828 \\
\hline \multicolumn{7}{|l|}{ VIOLACEAE } \\
\hline Viola subdimidiata A. St.-Hil. & - & - & - & $\mathrm{x}$ & ET & FM 2085 \\
\hline \multicolumn{7}{|l|}{ VITACEAE } \\
\hline Cissus salutaris Kunth & - & $\mathrm{x}$ & $\mathrm{x}$ & - & LI & FM 620 \\
\hline \multicolumn{7}{|l|}{ VOCHYSIACEAE } \\
\hline Qualea jundiahy Warm. & M.Rara & $\mathrm{x}$ & $\mathrm{x}$ & - & AV & FM 838, 904 \\
\hline Vochysia tucanorum Mart. & - & - & - & $\mathrm{x}$ & $\mathrm{AV}$ & FM 925 \\
\hline \multicolumn{7}{|l|}{ XYRIDACEAE } \\
\hline Xyris asperula Mart. & - & $\mathrm{x}$ & - & - & ET & FM 900 \\
\hline \multicolumn{7}{|c|}{ Pteridófitas } \\
\hline \multicolumn{7}{|l|}{ CYATHEACEAE } \\
\hline Cyathea villosa Willd. & - & $\mathrm{x}$ & - & - & AV & FM 852 \\
\hline \multicolumn{7}{|l|}{ GLEICHENIACEAE } \\
\hline Sticherus penniger (Mart.) C.V. Morton & - & $\mathrm{x}$ & $\mathrm{x}$ & - & ET & FM 906 \\
\hline \multicolumn{7}{|l|}{ POLYPODIACEAE } \\
\hline Phlebodium pseudoaureum (Cav.) Lellinger & - & $\mathrm{x}$ & $\mathrm{x}$ & - & ER & FM 912 \\
\hline Polypodium catharinae Langsd. \& Fisch. & - & $\mathrm{x}$ & $\mathrm{x}$ & - & ET & FM 907 \\
\hline Polypodium latipes Langsd. \& Fisch. & - & $\mathrm{x}$ & $\mathrm{x}$ & - & ET & FM 811 \\
\hline \multicolumn{7}{|l|}{ PTERIDACEAE } \\
\hline Adiantum sp. & - & - & - & $\mathrm{x}$ & ET & FM 2091 \\
\hline Doryopteris ornithopus (Mett.) J. Sm. & - & - & $\mathrm{x}$ & - & ET & FM 810 \\
\hline \multicolumn{7}{|l|}{ SCHIZAEACEAE } \\
\hline Anemia raddiana Link & - & $\mathrm{x}$ & $\mathrm{x}$ & - & ET & FM 812 \\
\hline
\end{tabular}

Malvaceae (13 spp.), Bignoniaceae, Euphorbiaceae e Lamiaceae (12 spp.), Apocynaceae, Malpighiaceae e Myrtaceae (11 spp.) (Tabela 2). Juntas, essas famílias perfazem $70 \%$ da riqueza de espécies amostradas na área.

O hábito mais frequente na área de estudo foi o herbáceo, em especial ervas terrestres (116 spp.), seguido por subarbustos (98 spp.), árvores (87 spp.), arbustos (63 spp.) e lianas (27 spp.) (Figura 3). As ervas escandentes foram representadas apenas por quatro espécies, todas associadas ao fragmento de floresta. Plantas que apresentam tal hábito, especialmente os bambus dos gêneros Merostachys e Chusquea, desempenham grande influência na dinâmica sucessional de ambientes florestais, tanto no aspecto qualitativo quanto quantitativo, devido ao crescimento vegetativo agressivo (Smith et al. 1981, Filgueiras 1988, Oliveira Filho et al. 1994b, Stern 1995) (Figura 2g). 


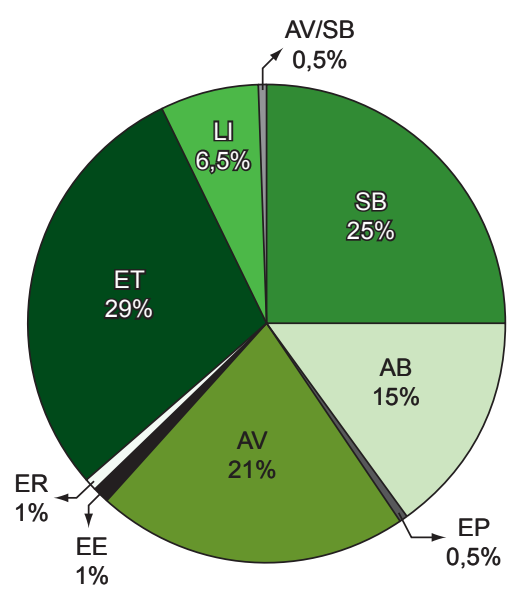

Figura 3. Porcentagem das espécies, com respectivos hábitos, inventariadas na Toca dos Urubus. AB: arbusto; AV: árvore; AV/SB: árvore/subarbusto; EE: erva escandente; EP: erva epífita; ER: erva rupícola; ET: erva terrestre; LI: liana; SB: subarbusto.

Figure 3. Species percentage, and life forms, found in the Toca dos Urubus. AB: shrub, AV: Tree, AV/SB: tree/subshrub, EE: clambering herb, EP: epiphyte, ER: rupicola herb, ET: herb, LI: vine, SB: subshrub.

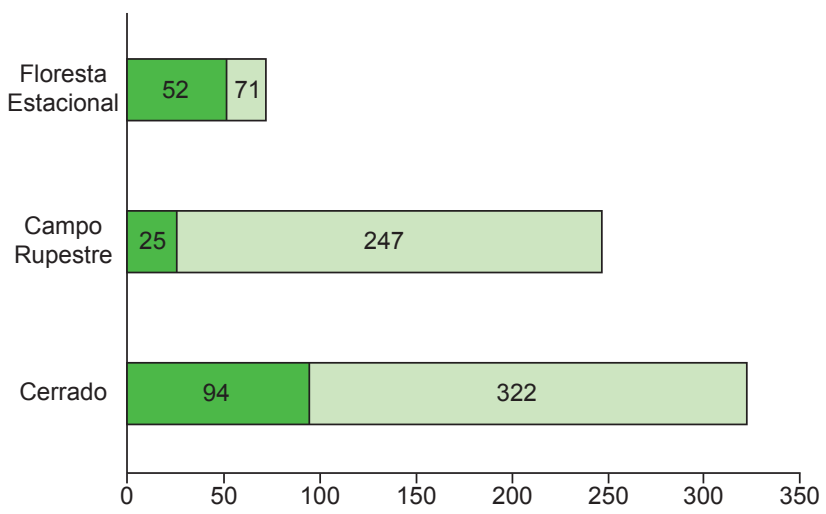

Figura 4. Número de espécies encontradas em cada uma das fisionomias da Toca dos Urubus. As barras escuras representam o número de espécies exclusivas para cada fisionomia.

Figure 4. Species richnes record on three physiognomies found in the Toca dos Urubus. The solid bars represent the exclusive species for each physiognomies.

Quatro espécies de ervas rupícolas (Barbacenia tomentosa, Peperomia oreophila, Phlebodium pseudoaureum e Phyllanthus rosellus) foram observadas somente no campo rupestre. Apenas duas espécies de epífitas foram encontradas, Polystachya estrellensis e Philodendron sp., a primeira encontrada no fragmento de mata e no cerrado e a segunda, observada apenas no interior da mata. Duas espécies, Andira fraxinifolia e Casearia sylvestris, são arbóreas, quando no interior da mata e subarbustivas nas formações campestres, o que é comum em espécies do cerrado sujeitas a contínua perda de suas partes aéreas (Rizzini 1979).

Para o cerrado, foram inventariadas 322 espécies, para o campo rupestre 247 e para a floresta estacional 71 . Dentre as espécies encontradas, 94 (ca. 23\%) foram exclusivas do cerrado, 52 (ca. 13\%) da floresta e apenas 25 (ca. 6,2\%) exclusivas do campo rupestre (Figura 4). O maior compartilhamento da flora foi observado entre o cerrado e o campo rupestre (213 spp., ca. 53\%) e o menor registrado entre o campo rupestre e a floresta (4 spp., ca. 0,9\%). Cerrado

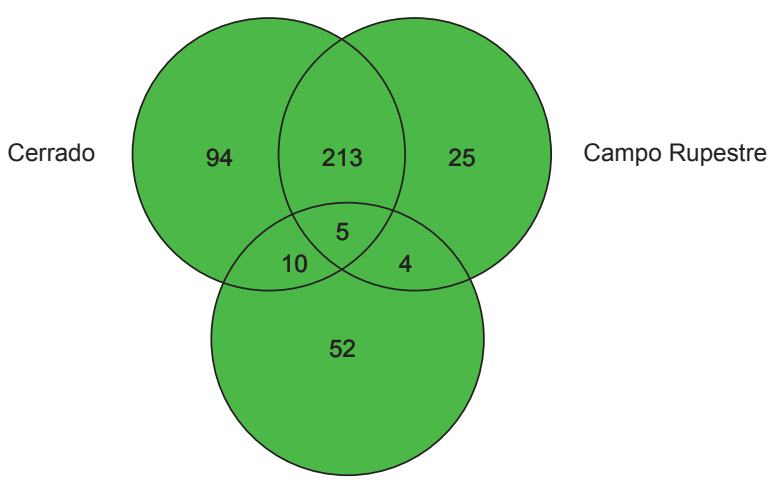

Floresta Estacional

Figura 5. Diagrama de Venn mostrando o número de espécies exclusivas e comuns entre as três fisionomias da Toca dos Urubus, Baependi.

Figure 5. Venn diagrams extracted from the checklists showing the number of species shared by three physiognomies in the Toca dos Urubus, Baependi.
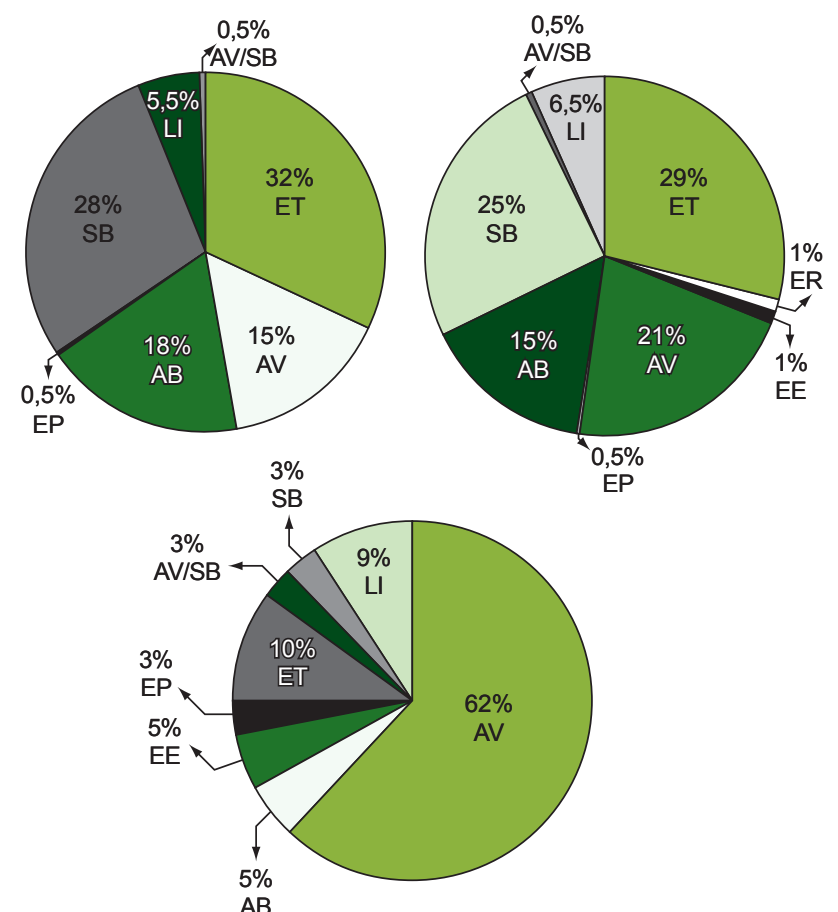

Figura 6. Percentagem de espécies, com respectivos hábitos, nas três fisionomias da Toca dos Urubus, Baependi. AB: arbusto; AV: árvore; AV/SB: árvore/ subarbusto; EE: erva escandente; EP: erva epífita; ER: erva rupícola; ET: erva terrestre; LI: liana; SB: subarbusto.

Figure 6. Species percentage, and life forms, on three physiognomies in the Toca dos Urubus, Baependi. AB: shrub, AV: Tree, AV/SB: tree/subshrub, EE: clambering herb, EP: epiphyte, ER: rupicola herb, ET: herb, LI: vine, SB: subshrub.

e floresta compartilharam 10 espécies (ca. 2,4\%) e cinco espécies ocorreram nas três formações (Figura 5).

De acordo com o Catálogo das Árvores Nativas de Minas Gerais (Oliveira Filho 2006), cinco espécies que ocorrem na Toca dos Urubus são raras (Gochnatia paniculata, Hyptidendron canum, Leandra aurea, Persea major e Psychothria spathicalix), cinco são citadas como muito raras (Aspidosperma parvifolium, Dasyphyllum 
Florística da Toca dos Urubus, Baependi

Tabela 2. Cinco famílias mais representativas na Toca dos Urubus (Geral) e em cada fisionomia

Table 2. Families with the highest number of species in Toca dos Urubus and, in the three vegetations formation.

\begin{tabular}{|c|c|c|c|c|c|c|c|}
\hline \multicolumn{2}{|l|}{ Geral } & \multicolumn{2}{|c|}{ Cerrado } & \multicolumn{2}{|c|}{ Campo Rupestre } & \multicolumn{2}{|c|}{ Floresta Estacional } \\
\hline Asteraceae & 66 & Asteraceae & 62 & Poaceae & 42 & Fabaceae & 10 \\
\hline Poaceae & 52 & Poaceae & 44 & Asteraceae & 23 & Euphorbiaceae & 8 \\
\hline Fabaceae & 32 & Fabaceae & 25 & Fabaceae & 17 & Poaceae & 6 \\
\hline Cyperaceae & 19 & Cyperaceae & 17 & Rubiaceae & 14 & Lauraceae & 3 \\
\hline Melastomataceae/Rubiaceae & 16 & Melastomataceae & 15 & Melastomataceae & 14 & Rubiaceae & 3 \\
\hline Total & 210 & Total & 163 & Total & 110 & Total & 30 \\
\hline
\end{tabular}

spinescens, Pseudobrickellia brasiliensis, Alchornea sidifolia e Qualea jundiahy) e uma como raríssima (Dalbergia glaucescens).

Dez espécies encontradas neste levantamento são citadas na Lista Vermelha das Espécies Ameaçadas de Extinção da Flora de Minas Gerais (Drummond et al. 2008). Dessas, uma está sob o status "Em Perigo" (Alstroemeria plantaginea); quatro são referidas como "Vulneráveis" (Chresta sphaerocephala, Ipomoea argenta, Ocotea odorifera, Camarea hyrsuta); uma é citada como "Quase Ameaçada" (Rhynchospora globosa), e quatro aparecem como "Dados Deficientes" (Chaetostoma inerme, Axonopus aureus, Axonopus brasiliensis e Chusquea tenuiglumis).

A fisionomia do cerrado, dominante na Toca dos Urubus, é formada por um estrato herbáceo, composto principalmente por gramíneas, e um estrato lenhoso um pouco esparso, formado por árvores de pequeno e médio porte com troncos retorcidos e escurecidos pelo fogo, e de arbustos de caules múltiplos (Figura 6). As famílias mais representativas dessa fisionomia foram Asteraceae (62 spp.), Poaceae (44 spp.), Fabaceae (25 spp.), Cyperaceae (17 spp.) e Melastomataceae (15 spp.) (Tabela 2).

Nessa formação, o estrato herbáceo é formado principalmente por ervas terrestres (103 spp., ca. 32\%) e subarbustos (91 spp., 28\%). Entre as ervas terrestres as famílias mais representativas foram Poaceae (44 spp.), Cyperaceae (17 spp.), Asteraceae (8 spp.) e Polygalaceae (6 spp.). A maioria dos subarbustos pertence às famílias Asteraceae (30 spp.), Fabaceae (13 spp.), Malvaceae (11 spp.), Rubiaceae (8 spp.) e Melastomataceae ( $7 \mathrm{spp}$.). O estrato lenhoso é composto por arbustos (57 spp., ca. 18\%) e árvores (51 spp., ca. 15\%), que corresponde a aproximadamente $33 \%$ da diversidade de espécies desse ambiente (Figura 6). As lianas correspondem a 5,5\% (18 spp.) da riqueza de espécies dessa formação.

O campo rupestre é um tipo de vegetação altamente especializada que ocorre como ilhas no alto de algumas serras brasileiras, normalmente acima dos $900 \mathrm{~m}$ de altitude e sobre solos muito rasos ou afloramentos rochosos (Harley 1995). Esses ambientes são reconhecidos por sua grande riqueza de espécies e endemismos (Joly 1970, Giulietti et al. 2000). Na Toca dos Urubus, os campos rupestres estão restritos à porção noroeste da serra e Poaceae (42 spp.), Asteraceae (23 spp.), Fabaceae (17 spp.), Melastomataceae e Rubiaceae (14 spp.) foram as famílias mais diversas desse ambiente (Tabela 2).

No campo rupestre, a paisagem é caracterizada pela grande quantidade de arbustos e um estrato herbáceo mais esparso do que o encontrado na área de cerrado. Dentre as ervas terrestres, principais componentes desse compartimento, destacaram-se as famílias Poaceae (42 spp.), Cyperaceae (16 spp.) e Asteraceae (6 spp.). Apesar de espécies rupícolas serem marcantes na composição dos campos rupestres, apenas quatro foram registradas (Tabela 1). Os subarbustos (59 spp., 24\%) são, em sua maioria, representados por espécies de Rubiaceae (10 spp.), Fabaceae (9 spp.), Asteraceae, Malvaceae e Melastomataceae (7 spp.).
Apesar da grande profusão de afloramentos rochosos e da pouca profundidade ou ausência do solo, cerca de $30 \%$ das espécies ocorrentes no campo rupestre foram de arbustos (41 spp., 16,5\%) e árvores (35 spp., 14\%), a maioria compartilhada com o cerrado (Figura 5, 6). Neste estrato, as famílias mais ricas em número de espécies foram Asteraceae (10 spp., 7 arbustos e 3 árvores), Lamiaceae (9 spp., 6 arbustos e 3 árvores), Myrtaceae (8 spp., 3 arbustos e 5 árvores), Melastomataceae (7 spp., 1 arbusto e 6 árvores) e Fabaceae (6 spp., 2 arbustos e 4 árvores). As lianas, com 18 espécies, corresponderam à aproximadamente $8 \%$ da riqueza de espécies do campo rupestre, e encontram-se intercaladas ao estrato graminoso e/ou sobre os arbustos e árvores.

A área de floresta estacional semidecidual está localizada no fundo de um vale adjacente ao curso d'água na vertente leste da serra. O fragmento é composto por quatro diferentes estratos: o dossel, que atinge entre 15 e $20 \mathrm{~m}$ de altura, o estrato médio entre 8 a $10 \mathrm{~m}$, o sub-bosque formado por arbustos e indivíduos jovens de árvores do dossel e sub-dossel e o estrato herbáceo. Neste ambiente, as famílias com maior número de espécies foram Fabaceae (10 spp.), Euphorbiaceae (8 spp.), Poaceae (6 spp.), Lauraceae e Rubiaceae (3 spp.) (Tabela 2). As espécies arbóreas foram maioria, cerca de $62 \%$ (44 spp.) (Figura 6). Lianas (6 spp., ca. 9\%) foram observadas no dossel, estrato médio, em clareiras e na borda da mata. As únicas espécies epífitas registradas foram Philodendron sp. e Polystachya estrellensis. As ervas mais comuns no interior da floresta foram Dichorisandra hexandra, Rhynchospora exaltata, Scleria bracteata e Chusquea breviglumis, que forma um denso taquaral na área próxima ao curso d'água e cobrindo grande parte do fragmento (Figura 2g).

Chama atenção o grande número de espécies ruderais e de ampla distribuição (55 spp.) que ocorrem na Toca dos Urubus (Tabela 1), além da perda constante da cobertura vegetal original (Figura 2d).

\section{Discussão}

Um dos fatores determinantes da diversidade de uma área é a estrutura da vegetação (Ricklefs 2003). A grande diversidade de espécies encontrada na Toca dos Urubus se deve, provavelmente, à riqueza fitofisionômica do local, composta por três formações principais: cerrado, campo rupestre e floresta estacional. Além disso, a transição entre tais fisionomias se dá de forma gradativa, constituindo gradientes vegetacionais que contribuem para o aumento da diversidade.

No presente estudo, a área de cerrado apresentou uma maior diversidade de espécies arbustivo-herbáceas em relação às arbóreas, conferindo uma proporção de aproximadamente 4,4:1. Tais resultados corroboram dados obtidos na Chapada Pratinha, Distrito Federal (Felfili et al. 1993), onde foi observada uma proporção de 4,5:1, porém diferente dos resultados apresentados por Mendonça et al. (1998), cuja razão foi de 3:1. A fisionomia do cerrado pode, de fato, apresentar variações em sua composição, principalmente relacionadas 
à proporção entre elementos lenhosos (árvores e arbustos) e herbáceos (Oliveira Filho \& Ratter 1995).

A grande diversidade de Asteraceae, Poaceae, Fabaceae, Cyperaceae e Melastomataceae é comum em áreas de cerrado, especialmente no que diz respeito às três primeiras, que estão entre as mais ricas para flora deste bioma (Mendonça et al. 1998). Essas famílias também constituíram os principais componentes do estrato herbáceosubarbustivo. Asteraceae, Fabaceae, Lamiaceae, Melastomataceae e Myrtaceae foram as famílias mais ricas em espécies formadoras do compartimento lenhoso dessa fisionomia. Malpighiaceae e Rubiaceae, duas famílias apontadas como importantes para esta fisionomia (Heringer et al. 1977) não estão entre as famílias mais diversas na Toca dos Urubus. A despeito disto, Byrsonima coccolobifolia e B. intermedia (Malpighiaceae), Palicourea rigida e Tocoyena formosa (Rubiaceae) são as espécies mais importantes do estrato arbustivo-arbóreo.

Para a flora do Cerrado s.1., Asteraceae figura entre as famílias mais diversas na composição do estrato herbáceo (Filgueiras 2002), sendo pouco representada no compartimento lenhoso (e.g. Batalha et al. 2001, Ratter et al. 2003, Pereira-Filho et al. 2004). Entretanto, esta foi uma das famílias mais representativas nesse estrato no cerrado da Toca dos Urubus, que pode estar associado ao fato de Asteraceae ser muito rica em espécies nas comunidades campestres do cerrado em Minas Gerais (Andrade et al. 1986).

O campo rupestre na Toca dos Urubus apresentou baixo número de espécies exclusivas, o que pode ser explicado pela pequena área ocupada por esta fisionomia. Tal fato contrasta com o que é esperado para áreas de campo rupestre que, no geral, são caracterizadas pelo grande número de espécies endêmicas (Giulietti et al. 2000). Fatores como atividades agropecuárias realizadas no passado, retirada de cascalho e a abertura de trilhas tanto por pessoas quanto pelo gado, certamente contribuíram para baixo número de táxons exclusivos encontrados. As famílias mais ricas dessa formação na área em estudo (Poaceae, Asteraceae e Fabaceae) são citadas entre as mais importantes em número de espécies para a flora dos campos rupestres (Joly 1970, Giulietti et al. 2000, Filgueiras 2002). Porém, vale ressaltar que famílias altamente diversas nos campos rupestres da Cadeia do Espinhaço (Eriocaulaceae, Xyridaceae e Velloziaceae) são pobremente representadas na Toca dos Urubus. A baixa diversidade destes grupos parece ser comum em outras áreas campestres da Serra da Mantiqueira (Forzza obs pess.).

A riqueza de espécies registrada na floresta estacional foi baixa em relação a outros estudos realizados na região do Alto Rio Grande (e.g. Oliveira Filho et al. 1994a, b, c, d, 2004; Rodrigues et al. 2003; Souza et al. 2003; Vilela et al. 1995, 2000). A retirada de parte da cobertura florestal original para a constituição de pastagens, certamente, reduziu significativamente a diversidade desse ambiente. Entretanto, o número de espécies catalogadas, encontra-se dentro dos limites esperados para florestas semidecíduas neotropicais (Gentry 1995). Fabaceae e Euphorbiaceae foram as famílias mais importantes em número de táxons arbustivo-arbóreos nesse ambiente, estando de acordo com o que é observado para florestas estacionais na Região Sudeste (Oliveira Filho et al. 2006). Apesar do pequeno número de espécies, o perfil florístico observado nessa formação na Toca dos Urubus é típico de florestas semideciduais montanas (Oliveira Filho et al. 1994b, 2006), sendo representado, principalmente, por Cariniana estrellensis, Casearia lasiophylla, Guatteria australis, Lamanonia ternata, Machaerium villosum, Ocotea odorifera, Protium widgrenii e Vitex polygama.

A flora da Toca dos Urubus mostrou-se bastante diferenciada entre os ambientes campestres e o florestal, resultando em uma grande riqueza local. O maior compartilhamento de espécies entre o cerrado e o campo rupestre pode ser explicado pela presença de um gradiente de transição entre essas fisionomias. A existência de áreas contíguas permite que espécies com alto potencial de dispersão possam ocupar mais de uma fisionomia (Costa \& Araujo 2001).

O grande número de espécies ruderais pode ser explicado pela presença de extensas áreas de pasto adjacentes ao local de estudo. Da mesma forma, a ocorrência de queimadas periódicas para manter as pastagens, prática comum em toda a região da APA Mantiqueira, contribui para o aparecimento dessas espécies generalistas que, em sua maioria, apresentam vantagens adaptativas para a colonização de áreas impactadas. Outra atividade realizada na área é a retirada de cascalho para a pavimentação de vias que cortam a região, o que facilita o aparecimento de ravinamentos e até mesmo voçorocas em consequência da retirada da cobertura vegetal.

Como já enfatizado por Azevedo (1962), a região dos altos divisores ou Alto Rio Grande é de extrema importância para a conservação e manutenção da bacia do Rio Paraguai e, consequentemente, do Rio da Prata. Tal afirmativa foi ressaltada recentemente no Atlas para a conservação da flora de Minas Gerais, onde esta região é tratada sob a categoria de importância biológica extrema, um dos níveis mais altos adotados (Drummond et al. 2005). A despeito disto, do apelo turístico e da riqueza vegetacional registrada no presente estudo, a região da Toca dos Urubus não conta com nenhuma unidade de conservação, o que se faz extremamente necessário para a manutenção e preservação das espécies e da vegetação aí ocorrentes.

O registro de quatro espécies indicadas como "Dados Deficientes" e seis ameaçadas de extinção da flora de Minas Gerais (Drummond et al. 2008), demonstra que levantamentos florísticos de longo prazo e que contam com a participação de especialistas representam fontes primárias e imprescindíveis para gerar informações para ações conservacionistas.

\section{Agradecimentos}

Os autores agradecem ao Sr. Cacildo Ferreira e ao pesquisador Régis M. Ferreira por todo incentivo e apoio nas campanhas de campo; à pesquisadora Ana Luísa de Carvalho Lima e a dois assessores anônimos pela leitura crítica do manuscrito; à Fátima R. Salimena, curadora do herbário CESJ, pelo apoio ao projeto; e aos especialistas que contribuíram na identificação das espécies: A. M. Teles, R. A. X. Borges, M. Saavedra e R. L. Esteves (Asteraceae), J. G. Jardim (Rubiaceae), M. Sobral (Myrtaceae), E. F. Guimarães (Boraginaceae, Gentianaceae e Piperaceae), L. Menini Neto (Orchidaceae), A. M. Amorim (Malpighiaceae), R. Goldenberg e B. Chiavegatto (Melastomataceae), R. Mello-Silva (Annonaceae e Velloziaceae), T. U. Kono e A. Rapini (Apocynaceae), V. Mansano (Fabaceae), R. Marquete (Salicaceae), A. Salino (Pteridófitas), L. C. S. Assis (Lauraceae), M. G. Bovini (Malvaceae), M. C. M. Marques (Polygalaceae), M. Trovó (Eriocaulaceae), R. M. Harley (Lamiaceae), R. M. Castro (Moraceae, Urticaceae), R. H. P. Andreata (Smilacaceae), F. R. Salimena (Verbenaceae) e F. França (Vochysiaceae). R. C. Forzza agradece ao CNPq pela bolsa de produtividade.

Monografia de conclusão de curso de Fabrício Moreira Ferreira desenvolvida na Universidade Federal de Juiz de Fora.

\section{Referências Bibliográficas}

ANDRADE, P.M., GONTIJO, T.A. \& GRANDI, T.S.M. 1986. Composição florística e aspectos estruturais de uma área de "campo rupestre" do morro do Chapéu, Nova Lima, Minas Gerais. Rev. Bras. Bot. 9(1):13-21.

AZEVEDO, L.G. 1962. Tipos de vegetação do sul de Minas e campos da Mantiqueira (Brasil). An. Acad. Bras. Cienc. 34(2):225-234.

BATALHA, M.A., MANTOVANIA, W. \& MESQUITA Jr., H.N. 2001. Vegetation structure in cerrado physiognomies in southeastern Brazil. Brazil. J. Biol. 61(3):475-483. 
Van den BERG, E. \& OLIVEIRA-FILHO, A.T. 2000. Composição florística e estrutura fitossociológica de uma floresta ripária em Itutinga, MG, e comparação com outras áreas. Rev. Brasil. Bot. 23(3):231-253.

BRAGA, F.M.S. \& ANDRADE, P.M. 2005. Distribuição de peixes na microbacia do Ribeirão Grande, Serra da Mantiqueira Oriental, São Paulo, Brasil. Iheringia, Sér. Zool. 95(2):121-126.

BOTREL, R.T., OLIVEIRA-FILHO, A.T., RODRIGUES, L.A. \& CURI, N. 2002. Influência do solo e topografia sobre as variações da composição florística e estrutura da comunidade arbóreo-arbustiva de uma floresta estacional semidecidual em Ingaí, MG. Rev. Brasil. Bot. 25(2):195-213.

CARVALHO, D.A., OLIVEIRA-FILHO, A.T., VILELA, E.A. \& GAVILANES, M.L. 1995. Flora arbustivo-arbórea de uma mata ciliar do Alto Rio Grande em Bom Sucesso - MG. Acta Bot. Brasil. 9(1):231-245.

COSTA, A.A. \& ARAÚJO, G.M. 2001. Comparação arbórea de cerrado e cerradão na Reserva do Panga, Uberlândia, MG. Acta Bot. Bras. 15(1):63-72.

DRUMMOND, G.M., MARTINS, C.S., MACHADO, A.B.M., SEBAIO, F.A. \& ANTONINI, Y. 2005. Biodiversidade em Minas Gerais: um atlas para sua conservação. 2 ed. Fundação Biodiversitas, Belo Horizonte.

DRUMMOND, G.M., MACHADO, A.B.M., MARTINS, C.S., MENDONÇA, M.P. \& STEHMANN, J.R. 2008. Listas vermelhas das espécies da fauna e flora ameaçadas de extinção em Minas Gerais. Fundação Biodiversitas, Belo Horizonte.

EITEN, G. 1982. The cerrado vegetation of Brazil. Bot. Review. 38(2):201-341

FELFILI, J.M., SILVA Jr., M.C., REZENDE, A.V., MACHADO, J.W.B., WALTER, B.M.T., SILVA, P.E.N. \& HAY, J.D. 1993. Análise comparativa da florística e fitossociologia da vegetação arbórea do cerrado sensu stricto na Chapada Pratinha, DF-Brasil. Acta Bot. Bras. 6(2):27-47.

FILGUEIRAS, T.S. 1988. A floração dos bambus e seu impacto ecológico. Eugeniana 15:1-8.

FILGUEIRAS, T.S. 2002. Herbaceous plant communities. In The cerrados of Brazil (P.S. Oliveira \& R.J. Marquis, eds.) New York, Columbia University Press, p. 121-139.

GAVILANES, M.L., BRANDÃO, M., OLIVEIRA-FILHO, A.T., ALMEIDA, R.J., MELLO, J.M. \& AVEZUM, F.F. 1992a. Flórula da Reserva Biológica Municipal do Poço Bonito, Lavras, MG. III Formação florestal. Daphne. 2(3):14-26.

GAVILANES, M.L., OLIVEIRA-FILHO, A.T., CARVALHO, D.A. \& VILELA, E.A. 1992b. Flora arbustivo-arbórea de uma mata ciliar do Alto Rio Grande em Madre de Deus de Minas - MG. Daphne. 2(4):15-24.

GENTRY, A.H. 1995. Diversity and floristic compositon of neotropical dry forests. In Seasonally dry tropical forests (S.H. Bullock, H.A. Money \& E. Medina, eds.). Cambridge University Press, Cambridge, p. 146-194.

GIULIETTI, A.M., MENEZES, N.L., PIRANI, J.R., MEGURO, M. \& WANDERLEY, M.G.L. 1987. Flora da Serra do Cipó, Minas Gerais: caracterização e lista das espécies. Bol. Bot. Univ. São Paulo. 9:1-151.

GIULIETTI, A.M., HARLEY. R.M., QUEIROZ, L.P., WANDERLEY, M.G.L., PIRANI, J.R. 2000. Caracterização e endemismos nos campos rupestres da Cadeia do Espinhaço. In Tópicos atuais de botânica (T.B. Cavalcanti \& B.M.T. Walter, eds.). EMBRAPA Recursos Genéticos, Brasília, p. 311-318.

GOMES, M.M. 2005. Diagnóstico sócio-econômico do Corredor Ecológico da Mantiqueira. Valor Natural, Belo Horizonte. (Relatório técnico)

HARLEY, R.M. 1995. Introduction. In Flora of the Pico das Almas, Chapada Diamantina, Bahia, Brazil (B. Stannard, ed.). Royal Botanic Gardens, Richmond, p. 1-78.

HERINGER, E.P., BARROSO, G.M., RIZZO, J.A. \& RIZZINI, C.T. 1977. A flora do cerrado. In Anais do IV Simpósio sobre o Cerrado. (M.G. Férri, org.). Editora da Universidade de São Paulo, São Paulo, p. 211-232.

HOLMGREN, P.K., HOLMGREN, N.H. \& BARNETT, L.C. 1990. Index Herbariorum: the herbaria of the world. New York Botanical Garden, New York.
Instituto Brasileiro de Geografia e Estatística - IBGE. 1993. Mapa da vegetação do Brasil. 1:5.000.000. IBGE, Rio de Janeiro.

JOLY, A.B. 1970. Conheça a vegetação brasileira. EDUSP; Polígono, São Paulo.

LORENZI, H. 1991. Plantas daninhas do Brasil: terrestres, aquáticas, parasitas, tóxicas e medicinais. 2 ed. Ed. Plantarum, Nova Odessa.

MENDONÇA, R.C., FELFILI, J.M., SILVA Jr., M.C., REZENDE, A.V., FILGUEIRAS, T.S. \& NOGUEIRA, P.E. 1998. Flora vascular do Cerrado. In Cerrado: ambiente e flora (S.M. Sano \& S.P. Almeida, orgs.) EMBRAPA; CPAC, Planaltina, p. 289-556.

OLIVEIRA-FILHO, A.T. 2006. Catálogo das árvores nativas de Minas Gerais: mapeamento e inventário da flora nativa e dos reflorestamentos de Minas Gerais. Editora UFLA, Lavras.

OLIVEIRA-FILHO, A.T. \& FLUMINHAN-FILHO, M. 1999. Ecologia da vegetação do Parque Florestal Quedas do Rio Bonito. Cerne. 5(2):51-64

OLIVEIRA-FILHO, A.T., VILELA, E.A., GAVILANES, M.L. \& CARVALHO, D.A. 1994a. Comparison of the Woody flora and soils of six areas of montane semideciduous forest in southern Minas Gerais, Brazil. Edinb. J. Bot. 51(3):355-389.

OLIVEIRA-FILHO, A.T., VILELA, E.A., GAVILANTES, M.L. \& CARVALHO, D.A. 1994b. Effect of flooding regime and understorey bamboos on the physiognomy and tree species composition of tropical semideciduous forest in Southeastern Brazil. Vegetatio. 113(2):99-124.

OLIVEIRA-FILHO, A.T., ALMEIDA, R.J., MELLO, J.M. \& GAVILANES, M.L. 1994c. Estrutura fitossociológica e variáveis ambientais em um trecho da mata ciliar do córrego dos Vilas Boas, Reserva Biológica do Poço Bonito, Lavras (MG). Rev. Brasil. Bot. 17(1):67-85.

OLIVEIRA-FILHO, A.T., SCOLFORO, J.R. \& MELLO, J.M. 1994d. Composição florística e estrutura comunitária de um remanescente de floresta semidecídua montana em Lavras (MG). Rev. Brasil. Bot. 17(2):159-174.

OLIVEIRA-FILHO, A.T. \& RATTER, J.A. 1995. A study of the origin of central Brazilian forests by the analysis of plant species distribution patterns. Edinb. J. Bot. 52(2):141-194.

OLIVEIRA-FILHO, A.T., CARVALHO, D.A., FONTES, M.A.L., Van den BERG, E., CURI, N. \& CARVALHO, W.A.C. 2004. Variações estruturais do compartimento arbóreo de uma floresta semidecídua alto-montana na chapada das Perdizes, Carrancas, MG. Rev. Brasil. Bot. 27(2):291-309.

OLIVERIRA-FILHO, A.T., JARENKOW, J.A. \& RODAL, M.J.N. 2006. Floristic relationships of seasonally dry forests of eastern South America based on tree species distribution patterns. In Neotropical savannas and seasonally dry forests: plant diversity, biogeography, and conservation (R.T. Pennington, G.P. Lewis \& J. Ratter, eds.). Taylor \& Francis CRC Press, Oxford, p. 59-192.

PEREIRA-FILHO, E.F.L., SANTOS, J.E., KAGEYAMA, P. \& HARDT, E. 2004. Florística e fitossociologia dos estratos arbustivos e arbóreo de um remanescente de cerradão em uma Unidade de Conservação do Estado de São Paulo. Rev. Bras. Bot. 27(3):533-544.

PONÇANO, W.L., CARNEIRO, C.D.R., BISTRICHI, C.A., ALMEIDA, F.F.M. \& PRANDINI, F.L. 1981. Mapa Geomorfológico do Estado de São Paulo. Instituto de Pesquisas Tecnológicas, São Paulo.

RATTER, J.A., BRIDGEWATER, S. \& RIBEIRO, J.F. 2003. Analysis of floristic composition of the Brazilian cerrado vegetation. III comparison of the woody vegetation of 376 areas. Edinb. J. Bot. 60(1):57-109.

RIBEIRO, K.O. 2005. Ação coletiva, conselho consultivo e gestão: um estudo da área de proteção ambiental Serra da Mantiqueira. Dissertação de Mestrado, Universidade Federal de Lavras, Lavras.

RICKLEFS, R.E. 2003. Economia da natureza. 5 ed. Editora Guanabara Koogan, Rio de Janeiro.

RIZZINI, C.T. 1979. Tratado de fitogeografia do Brasil: aspectos sociológicos e florísticos. HUCITEC; EDUSP, São Paulo.

RODRIGUES, L.A., CARVALHO, D.A., OLIVEIRA-FILHO, A.T., BOTREL, R.T. \& SILVA, E.A. 2003. Florística e estrutura da comunidade 
arbórea de um fragmento florestal em Luminárias, MG. Acta Bot. Brasil. 17(1):71-87.

Secretaria de Estado de Ciência e Tecnologia. 1982. Atlas Geoeconômico da Microrregião do Circuito das Águas. Instituto de Geociências Aplicadas, Belo Horizonte.

SILVA, B.S. 2005. A experiência do SIG e sensoriamento remoto na construção de um gerenciamento participativo na Serra da Mantiqueira. In Anais do XII Simpósio Brasileiro de Sensoriamento Remoto. Instituto Nacional de Pesquisas Espaciais, São José dos Campos, p. 1375-1380. http://marte. dpi.inpe.br/col/ltid.inpe.br/sbsr/2004/11.22.20.37/doc/1375.pdf (último acesso em: 24/03/2009).

SMITH, L.B., WASSHAUSEN, D.C. \& KLEIN, R.M. 1981. Gramíneas. In Flora ilustrada catarinense (R. Reitz, ed.). Herbário Barbosa Rodrigues, Itajaí, p. 1-442. (v. 1).

SOUZA, J.S., ESPÍRITO-SANTO, F.D.B., FONTES, M.A.L., OLIVEIRAFILHO, A. T. \& BOTEZELLI, L. 2003. Análise das variações florísticas e estruturais da comunidade arbórea de um fragmento de floresta semidecídua às margens do rio Capivari, Lavras, MG. Rev. Árvore. 27(2):1-22

STERN, M.J. 1995. Vegetation recovery on earthquake-triggered landslide sites in the Ecuadorian Andes. In Biodiversity and conservation of neotropical montane forests (S.P. Churchill, H. Balslev, E. Forero \& J. Luteyn, eds.). New York Botanical Garden, Bronx, p. 207-220.

VELOSO, H.P., RANGEL-FILHO, A.L.R. \& LIMA, J.C.A. 1991. Classificação da vegetação brasileira, adaptada a um sistema universal. IBGE, Rio de Janeiro.

VILELA, E.A., OLIVEIRA-FILHO, A.T., CARVALHO, D.A. \& GAVILANES, M.L. 1995. Flora arbustivo-arbórea de um fragmento de mata ciliar no Alto Rio Grande, Itutinga, Minas Gerais. Acta Bot. Brasil. 9(1):87-100.

VILELA, E.A., OLIVEIRA-FILHO, A.T., CARVALHO, D.A., GUILHERME, F.A.G. \& APPOLINÁRIO, V. 2000. Caracterização estrutural de floresta ripária do Alto Rio Grande, em Madre de Deus de Minas, MG. Cerne. 6(2):41-54.

VIOLA, M.R. 2008. Simulação hidrológica na região Alto Rio Grande a montante do Reservatório de Camargos. Dissertação de Mestrado, Universidade Federal de Lavras, Lavras.

Recebido em 20/05/09

Versão reformulada recebida em 04/09/09

Publicado em 07/09/09 